\title{
Lipases in $\beta$-Dipeptide Synthesis in Organic Solvents
}

\author{
Xiang-Guo Li and Liisa T. Kanerva* \\ Department of Pharmacology, Drug Development and Therapeutics/Laboratory of Synthetic Drug \\ Chemistry and Department of Chemistry \\ University of Turku, Lemminkaisenkatu 5 C, FIN-20520, Turku, Finland \\ lkanerva@utu.fi
}

\section{Supporting Information}


Materials and methods. 2,2,2-Trifluoroethyl butanoate was prepared from butanoyl chloride and 2,2,2-trifluoroethanol. 3-Aminopropanoate esters $\mathbf{1 a}$ and 1e were prepared from $\beta$-alanine by esterification in the presence of thionyl chloride and an alcohol. Esters $\mathbf{1 b}$-d and their enantiomers were obtained as described earlier. ${ }^{1-3} \mathrm{~N}$-Acylations of $\mathbf{1 a}$-d with acetic anhydride in the presence of triethylamine in dichloromethane yielded 2a-d. All solvents were of the highest analytical grade and were dried by standard methods when necessary. CAL-A (lipase A from Candida antarctica, Chirazyme L5, lyo.) was purchased from Boehringer-Mannheim. Before use, CAL-A was adsorbed on celite by dissolving the enzyme $(5 \mathrm{~g})$ and sucrose $(3 \mathrm{~g})$ in Tris- $\mathrm{HCl}$ buffer $(250 \mathrm{~mL}, 20 \mathrm{mM}$, $\mathrm{pH}=7.9)$ followed by the addition of celite $(17 \mathrm{~g}) .{ }^{4}$ The lipase preparation [contains $20 \%(\mathrm{w} / \mathrm{w})$ of the original CAL-A powder] gave $v_{0}=0.086 \pm 0.001 \mathrm{mmol} / \mathrm{min} / \mathrm{g}$ and $E=13$ for the reaction of racemic valine methyl ester $(0.1 \mathrm{M})$ with 2,2,2-trifluoroethyl butanoate $(0.2 \mathrm{M})$ in TBME as a model reaction. CAL-B (lipase B from $C$. antarctica, Novozym 435) was a generous gift from Novo Nordisk.

Preparative chromatographic separations were performed by column chromatography on Merck Kieselgel $60(0.063-0.200 \mu \mathrm{m})$. TLC was carried out with Merck Kieselgel $60 \mathrm{~F}_{254}$ sheets. HPLC analysis was conducted with a HP 1090 instrument using a CHIRACEL OD column $(0.46 \times 25 \mathrm{~cm})$. Elemental analyses were performed with a Perkin-Elmer CHNS-2400 Ser II Elemental Analyser. Progress of reactions and ee values were followed by taking samples at intervals and analyzing them by gas chromatography on a Chrompack CP-Chirasil-L-Val column $(25 \mathrm{~m})$ or on a Chrompack CP-Chirasil-DEX CB column $(25 \mathrm{~m})$.

The ${ }^{1} \mathrm{H}$ and ${ }^{13} \mathrm{C}$ NMR spectra were recorded on a Bruker 500 spectrometer with tetramethylsilane (TMS) as an internal standard. ${ }^{1} \mathrm{H}-{ }^{1} \mathrm{H}$ COSY, ${ }^{1} \mathrm{H}_{-}{ }^{13} \mathrm{C}$ HSQC and ${ }^{1} \mathrm{H}_{-}{ }^{13} \mathrm{C}$ HMBC spectra were used for the assignment of chemical shifts in some cases. Mass spectra were taken on a VG 7070E mass spectrometer. Optical rotations were determined with a Perkin-Elemer polarimeter, and $[\alpha]_{D}{ }^{23}$ values $\left(c 1, \mathrm{CHCl}_{3}\right)$ are given in units of $10^{-1} \mathrm{deg} \mathrm{cm}^{2} \mathrm{~g}^{-1}$. Melting points were measured on a Sanyo instrument at a heating rate of $2{ }^{\circ} \mathrm{C} / \mathrm{min}$. If not otherwise stated, all enzymatic reactions were performed at room temperature $\left(23{ }^{\circ} \mathrm{C}\right)$.

Transformations of 2 into 2,2,2-trifluoroethyl esters 3

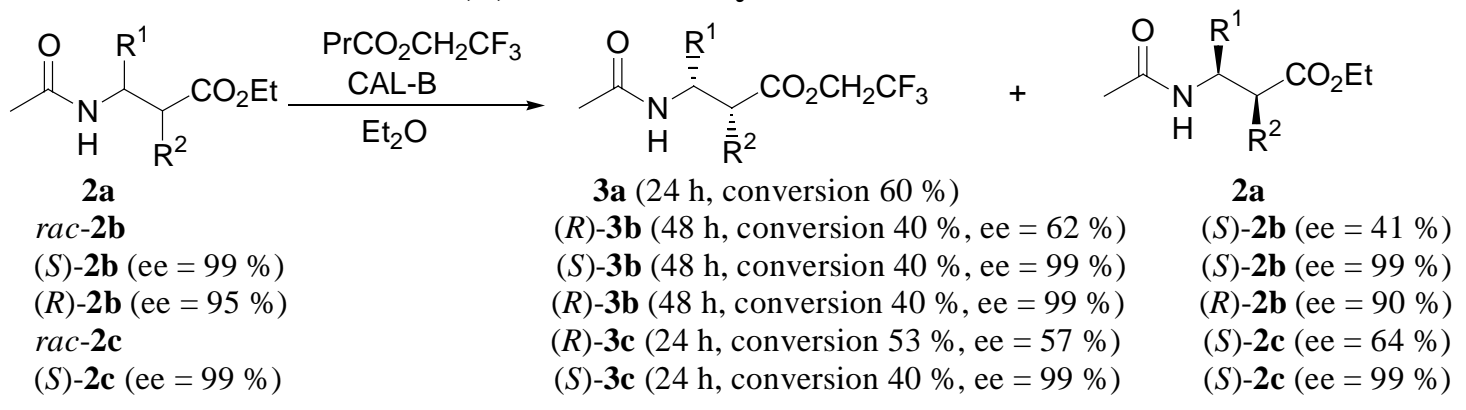

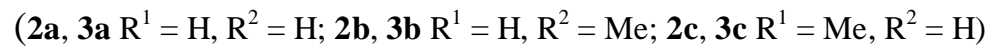

CAL-B (20 $\left.\mathrm{mg} \mathrm{mL}^{-1}\right)$ was added to a solution containing $\mathbf{2 a}$ or $\mathbf{2 c}(0.1 \mathrm{M})$ and 2,2,2-trifluoroethyl butanoate $(0.2 \mathrm{M})$ in dry diethyl ether. In the case of $\mathbf{2 b}$, CAL-B content was $40 \mathrm{mg} \mathrm{mL}^{-1}$. The reaction was stopped by filtering off the enzyme after 24-48 hours. The solvent and the excess of 2,2,2-trifluoroethyl butanoate were evaporated and the residue containing $\mathbf{3 a}, \mathbf{3 b}$ or $\mathbf{3 c}$ was used to dipeptide (4) synthesis without further purification.

\section{Methyl 3-(3-N-acetylaminopropanoyl)aminopropanoate 4a.}




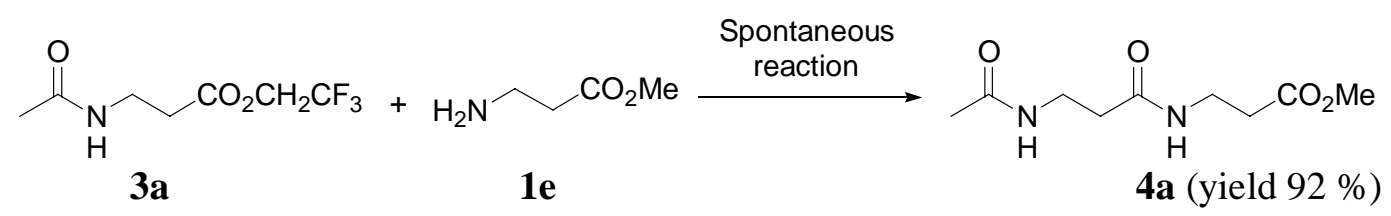

3a $(0.18 \mathrm{mmol}, 38 \mathrm{mg})$ and $\mathbf{1 e}(0.36 \mathrm{mmol}, 37 \mathrm{mg})$ were let to react in dry diethyl ether $(1.8 \mathrm{~mL})$. After 3a was consumed, the solvent was evaporated off and the residue was purified on silica gel using elution with methanol ( $3 \%, \mathrm{v} / \mathrm{v})$ in dichloromethane, affording $4 \mathbf{a}(0.17 \mathrm{mmol}, 35 \mathrm{mg})$. Isolated yield $92 \%$, white solid, m.p. $129-130{ }^{\circ} \mathrm{C}$; $\mathrm{HRMS} \mathrm{M}{ }^{+}$found $\left(\mathrm{M}^{+}\right.$calculated for $\left.\mathrm{C}_{9} \mathrm{H}_{16} \mathrm{~N}_{2} \mathrm{O}_{4}\right)$ : $216.11090(216.11101)$; MS: $m / z$ (relative intensity) $=216\left(\mathrm{M}^{+}, 50\right), 173(58), 126(41), 114(95)$, $102(61), 72(100) ;{ }^{1} \mathrm{H}$ NMR $\left(500 \mathrm{~Hz}, \mathrm{CDCl}_{3}\right): \delta=1.96(\mathrm{~s}, 3 \mathrm{H}), 2.40(\mathrm{t}, J=5.50 \mathrm{~Hz}, 2 \mathrm{H}), 2.55(\mathrm{t}, J$ $=6.00 \mathrm{~Hz}, 2 \mathrm{H}), 3.46-3.56(\mathrm{~m}, 4 \mathrm{H}), 3.70(\mathrm{~s}, 3 \mathrm{H}), 6.39(\mathrm{br} \mathrm{s}, 1 \mathrm{H}, \mathrm{NH}), 6.55(\mathrm{br} \mathrm{s}, 1 \mathrm{H}, \mathrm{NH}) ;{ }^{13} \mathrm{C}$ NMR $\left(126 \mathrm{~Hz}, \mathrm{CDCl}_{3}\right): \delta=23.24,33.79,34.94,35.45,35.52,51.93,170.54,171.76,172.90$; Elemental analysis for $\mathrm{C}_{9} \mathrm{H}_{16} \mathrm{~N}_{2} \mathrm{O}_{4}$ : found $\mathrm{C}, 50.23 \% ; \mathrm{H}, 7.40 \% ; 12.81 \%$, calcd. C, 49.99\%; $\mathrm{H}$, $7.46 \% ; \mathrm{N}, 12.96 \%$.

Ethyl 3-(3- $N$-acetylaminopropanoyl)amino-2-methylpropanoate $4 \mathrm{~b}$.

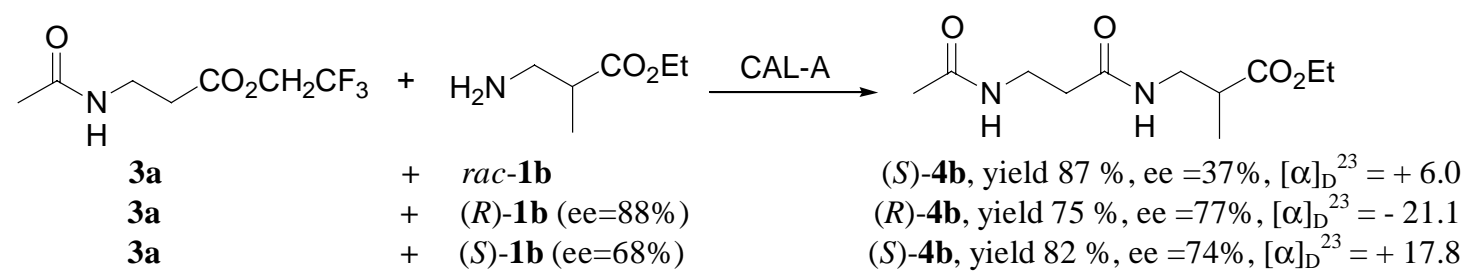

CAL-A preparation $\left(30 \mathrm{mg} \mathrm{mL}^{-1}\right)$ was added to a solution of $\mathbf{3 a}(0.18 \mathrm{mmol}, 38 \mathrm{mg})$ and $\mathrm{rac}-\mathbf{1} \mathbf{b}$ $(0.36 \mathrm{mmol}, 47 \mathrm{mg})$ in dry diethyl ether $(1.8 \mathrm{~mL})$. The reaction was stopped by filtering off the enzyme when 3a had reacted. The workup was done as above, affording $(S)-\mathbf{4 b}(0.16 \mathrm{mmol}, 39$ mg). Isolated yield $87 \%$, white solid, m.p. 108-109 ${ }^{\circ} \mathrm{C}$; HRMS $\mathrm{M}^{+}$found $\left(\mathrm{M}^{+}\right.$calculated for $\mathrm{C}_{11} \mathrm{H}_{20} \mathrm{~N}_{2} \mathrm{O}_{4}$ ): 244.14220 (244.14231); $\mathrm{MS}: \mathrm{m} / z$ (relative intensity) = $244\left(\mathrm{M}^{+}, 22\right), 199(15), 130$ (21), 114 (100), $72(53) ;{ }^{1} \mathrm{H}$ NMR $\left(500 \mathrm{~Hz}, \mathrm{CDCl}_{3}\right): \delta=1.19\left(\mathrm{~d}, J=7.00 \mathrm{~Hz}, 3 \mathrm{H}, \mathrm{CH}_{3} \mathrm{CH}\right), 1.28(\mathrm{t}$, $J=7.50 \mathrm{~Hz}, 3 \mathrm{H}, \mathrm{CH}_{3} \mathrm{CH}_{2}$ ), $1.96\left(\mathrm{~s}, 3 \mathrm{H}, \mathrm{CH}_{3} \mathrm{CO}\right), 2.40\left(\mathrm{t}, J=6.00 \mathrm{~Hz}, 2 \mathrm{H}, \mathrm{CH}_{2} \mathrm{CH}_{2} \mathrm{CO}\right.$ ), 2.64-2.70 ( m, $\left.1 \mathrm{H}, \mathrm{CH}_{2} \mathrm{CH}\right), 3.30-3.35\left(\mathrm{~m}, 1 \mathrm{H}, \mathrm{CH}_{2} \mathrm{CH}\right), 3.49-3.54\left(\mathrm{~m}, 3 \mathrm{H}, \mathrm{CH}_{2} \mathrm{CH}_{2} \mathrm{CO}\right.$ and $\left.\mathrm{CH}_{2} \mathrm{CH}\right), 4.13-$ $4.18\left(\mathrm{~m}, 2 \mathrm{H}, \mathrm{CH}_{2} \mathrm{CH}_{3}\right), 6.30($ br s, $1 \mathrm{H}, \mathrm{NH}), 6.53($ br s, $1 \mathrm{H}, \mathrm{NH}) ;{ }^{13} \mathrm{C} \mathrm{NMR}\left(126 \mathrm{~Hz}, \mathrm{CDCl}_{3}\right): \delta=$ $14.18\left(\mathrm{CH}_{3} \mathrm{CH}_{2}\right), 14.83\left(\mathrm{CH}_{3} \mathrm{CH}\right), 23.25\left(\mathrm{CH}_{3} \mathrm{CO}\right), 35.50,35.56,39.51\left(\mathrm{CH}_{3} \mathrm{CH}\right), 41.65,60.86$ $\left(\mathrm{CH}_{3} \mathrm{CH}_{2}\right)$, 170.41, 171.81, 175.37; Elemental analysis for $\mathrm{C}_{11} \mathrm{H}_{20} \mathrm{~N}_{2} \mathrm{O}_{4}$ : found $\mathrm{C}, 53.41 \%$; $\mathrm{H}$, $8.20 \%$; N, 10.79\%, calcd. C, 54.08\%; H, 8.25\%; N, $11.47 \%$.

Ethyl 3-(3-N-acetylaminopropanoyl)aminobutanoate 4c.

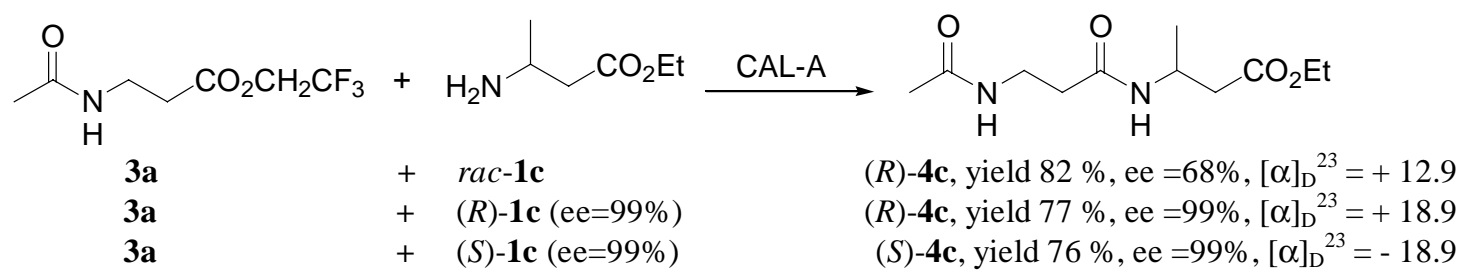

CAL-A preparation $(30 \mathrm{mg} / \mathrm{mL})$ was added to a solution of $\mathbf{3 a}(0.18 \mathrm{mmol}, 38 \mathrm{mg})$ and $\mathrm{rac}-\mathbf{1 c}(0.36$ $\mathrm{mmol}, 47 \mathrm{mg})$ in dry diethyl ether $(1.8 \mathrm{~mL})$. Following the above procedure $(R)-4 \mathrm{c}(0.15 \mathrm{mmol}, 37$ 
mg) was obtained at $82 \%$ isolated yield, white solid, m.p. $132-133{ }^{\circ} \mathrm{C}$; HRMS $\mathrm{M}^{+}$found $\left(\mathrm{M}^{+}\right.$ calculated for $\mathrm{C}_{11} \mathrm{H}_{20} \mathrm{~N}_{2} \mathrm{O}_{4}$ ): 244.14236 (244.14231); $\mathrm{MS}: \mathrm{m} / \mathrm{z}$ (relative intensity) $=244\left(\mathrm{M}^{+}, 15\right)$, 199 (17), 157 (13), 130 (78), 114 (100), 72 (56); ${ }^{1} \mathrm{H}$ NMR $\left(500 \mathrm{~Hz}, \mathrm{CDCl}_{3}\right): \delta=1.23$ ( d, $J=6.50$ $\mathrm{Hz}, 3 \mathrm{H}), 1.27(\mathrm{t}, J=7.00 \mathrm{~Hz}, 3 \mathrm{H}), 1.97(\mathrm{~s}, 3 \mathrm{H}), 2.36-2.40(\mathrm{~m}, 2 \mathrm{H}), 2.45-2.50(\mathrm{dd}, J=15.50 \mathrm{~Hz}$, $6.00 \mathrm{~Hz}, 1 \mathrm{H}), 2.51-2.55(\mathrm{dd}, J=15.50 \mathrm{~Hz}, 5.50 \mathrm{~Hz}, 1 \mathrm{H}), 3.47-3.57(\mathrm{~m}, 2 \mathrm{H}), 4.15(\mathrm{~m}, 2 \mathrm{H}), 4.29-$ $4.41(\mathrm{~m}, 1 \mathrm{H}), 6.37$ (br. s, $1 \mathrm{H}, \mathrm{NH}), 6.54$ (brs, $1 \mathrm{H}, \mathrm{NH}) ;{ }^{13} \mathrm{C} \mathrm{NMR}\left(126 \mathrm{~Hz}, \mathrm{CDCl}_{3}\right): \delta=14.18$, $20.13,23.22,35.60,35.71,40.18,42.29,60.76,170.55,170.98,171.67$; Elemental analysis for $\mathrm{C}_{11} \mathrm{H}_{20} \mathrm{~N}_{2} \mathrm{O}_{4}$ : found $\mathrm{C}, 53.95 \% ; \mathrm{H}, 8.37 \% ; \mathrm{N}, 10.98 \%$, calcd. C, $54.08 \% ; \mathrm{H}, 8.25 \% ; 11.47 \%$.

\section{3-(3-acetylaminopropanoyl)amino-3-phenylpropanoate $4 \mathrm{~d}$.}

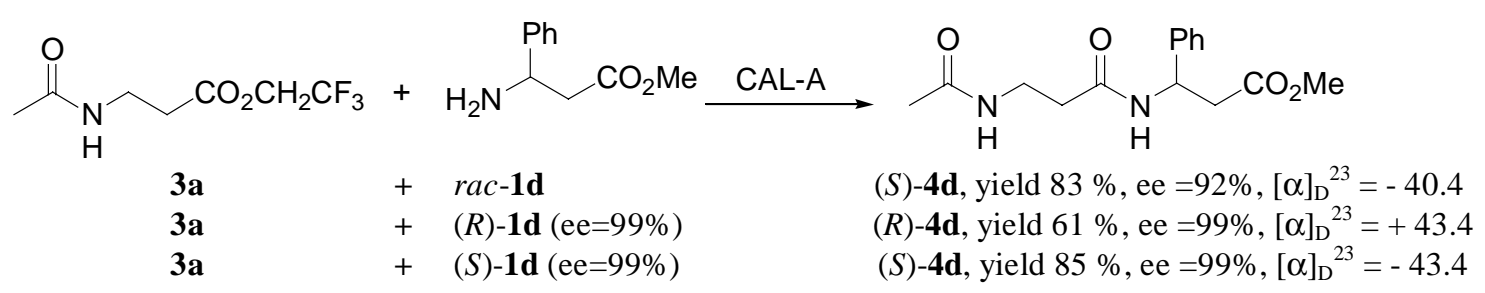

CAL-A preparation $\left(30 \mathrm{mg} \mathrm{mL}^{-1}\right)$ was added to a solution of $\mathbf{3 a}(0.18 \mathrm{mmol}, 38 \mathrm{mg})$ and $\mathrm{rac}-\mathbf{1 d}$ $(0.36 \mathrm{mmol}, 64 \mathrm{mg})$ in dry diethyl ether $(1.8 \mathrm{~mL})$. Following the above procedure $(S)-\mathbf{4 d}(0.15$ mmol, $44 \mathrm{mg}$ ) was obtained at $83 \%$ isolated yield, white solid, m.p. $124-125{ }^{\circ} \mathrm{C}$; HRMS $\mathrm{M}^{+}$found $\left(\mathrm{M}^{+}\right.$calculated for $\left.\mathrm{C}_{15} \mathrm{H}_{20} \mathrm{~N}_{2} \mathrm{O}_{4}\right): 292.14273$ (292.14231); $\mathrm{MS}: \mathrm{m} / z$ (relative intensity) = 292 (3), 279 (2), 219(4), 178 (100), 114 (23), 106 (57); ${ }^{1} \mathrm{H}$ NMR $\left(500 \mathrm{~Hz}, \mathrm{CDCl}_{3}\right): \delta=1.91$ (s, 3H, $\left.\mathrm{CH}_{3} \mathrm{CO}\right), 2.44\left(\mathrm{t}, J=6.0 \mathrm{~Hz}, 2 \mathrm{H}, \mathrm{CH}_{2} \mathrm{CH}_{2} \mathrm{CONH}\right), 2.80-2.90(\mathrm{dd}, J=15.5,7.0 \mathrm{~Hz}, 2 \mathrm{H}$, $\left.\mathrm{CHCH}_{2} \mathrm{CO}_{2}\right), 3.46-3.55\left(\mathrm{~m}, 2 \mathrm{H}, \mathrm{CH}_{2} \mathrm{CH}_{2} \mathrm{CONH}\right), 3.63\left(\mathrm{~s}, 3 \mathrm{H}, \mathrm{CO}_{2} \mathrm{CH}_{3}\right), 5.40(\mathrm{q}, J=6.8 \mathrm{~Hz}, 1 \mathrm{H}$, $\left.\mathrm{CHCH}_{2}\right), 7.26-7.30$ (m, 3H, aroma.), 7.32-7.35 (m, 2H, aroma.); ${ }^{13} \mathrm{C} \mathrm{NMR}\left(126 \mathrm{~Hz}, \mathrm{CDCl}_{3}\right): \delta=$ $23.21\left(\mathrm{CH}_{3}\right), 35.57\left(\mathrm{CH}_{2} \mathrm{CH}_{2}\right), 35.70\left(\mathrm{CH}_{2} \mathrm{CH}_{2}\right), 40.10\left(\mathrm{CHCH}_{2}\right), 49.89(\mathrm{CH}), 51.95\left(\mathrm{CH}_{3}\right), 126.24$, 127.83, 128.83, 140.47, 170.55, 171.03, 171.58; Elemental analysis for $\mathrm{C}_{15} \mathrm{H}_{20} \mathrm{~N}_{2} \mathrm{O}_{4}$ : found $\mathrm{C}$, $61.42 \%$; H, 6.88\%; 9.41\%, calcd. C, 61.63\%; H, 6.90\%; N, $9.58 \%$.

\section{Ethyl 3-(3- $N$-acetylamino-2-methylpropanoyl)aminopropanoate 4e.}

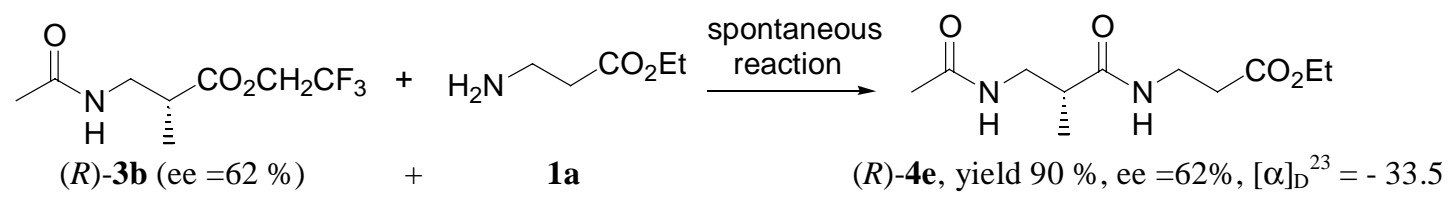

$(R)-3 \mathbf{b}(0.18 \mathrm{mmol}, 41 \mathrm{mg})$ and $\mathbf{1 a}(0.35 \mathrm{mmol}, 41 \mathrm{mg})$ were let to react in dry diethyl ether $(1.76$ $\mathrm{mL})$. Following the above procedure $(R)-4 \mathrm{e}\left(0.16 \mathrm{mmol}, 39 \mathrm{mg}\right.$, ee $\left.=62 \%,[\alpha]_{\mathrm{D}}=-33.5\right)$ was obtained at $90 \%$ isolated yield, white solid, m.p. $110-111^{\circ} \mathrm{C} ; \mathrm{HRMS} \mathrm{M}^{+}$found $\left(\mathrm{M}^{+}\right.$calculated for $\mathrm{C}_{11} \mathrm{H}_{20} \mathrm{~N}_{2} \mathrm{O}_{4}$ ): 244.14284 (244.14231); $\mathrm{MS}: \mathrm{m} / z$ (relative intensity) = $244\left(\mathrm{M}^{+}, 70\right), 199(40), 173$ (38), 140 (41), 118 (68), $58(100)$; ${ }^{1} \mathrm{H}$ NMR (500Hz, $\left.\mathrm{CDCl}_{3}\right): \delta=1.12(\mathrm{~d}, J=7.50,3 \mathrm{H}), 1.26$ ( t, $3 \mathrm{H}, J=7.00 \mathrm{~Hz}), 1.98(\mathrm{~s}, 3 \mathrm{H}), 2.49-2.54(\mathrm{~m}, 2 \mathrm{H}), 3.23-3.28(\mathrm{~m}, 1 \mathrm{H}), 3.39-3.59(\mathrm{~m}, 4 \mathrm{H}), 4.17$ ( q, $J=7.00 \mathrm{~Hz}, 2 \mathrm{H}), 6.51($ br s, $2 \mathrm{H}, \mathrm{N} H$ and $\mathrm{NH}) ;{ }^{13} \mathrm{C} \mathrm{NMR}\left(126 \mathrm{~Hz}, \mathrm{CDCl}_{3}\right): \delta=14.18,15.59,23.17$, $34.09,34.98,40.39,42.40,60.88,170.61,172.53,175.23$; Elemental analysis for $\mathrm{C}_{11} \mathrm{H}_{20} \mathrm{~N}_{2} \mathrm{O}_{4}$ : found C, 54.03\%; H, 8.24\%; N, 11.18\%, calcd. C, 54.08\%; H, 8.25\%; $11.47 \%$.

Methyl 3-(3- $N$-acetylamino-2-methylpropanoyl)amino-3-phenylpropanoate 4f. 


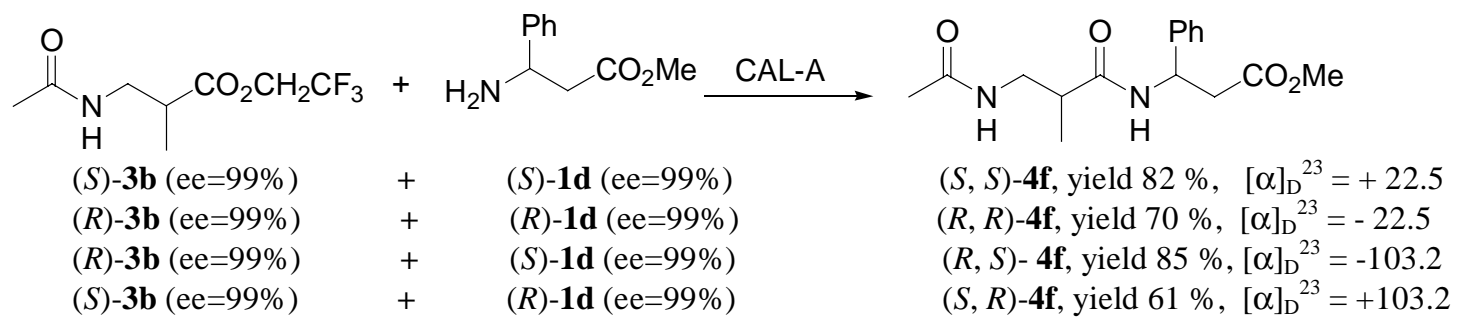

CAL-A preparation $\left(30 \mathrm{mg} \mathrm{mL}^{-1}\right)$ was added to a solution of $(S)-\mathbf{3 b}(0.18 \mathrm{mmol}, 41 \mathrm{mg})$ and $(S)$-1d $(0.36 \mathrm{mmol}, 64 \mathrm{mg})$ in dry diisopropyl ether $(1.8 \mathrm{~mL})$. Following the above procedure $(S, S)-\mathbf{4 f}$ $(0.15 \mathrm{mmol}, 45 \mathrm{mg})$ was obtained at $82 \%$ isolated yield, white solid, m.p. $174-175{ }^{\circ} \mathrm{C}$; $\mathrm{HRMS} \mathrm{M}^{+}$ found $\left(\mathrm{M}^{+}\right.$calculated for $\mathrm{C}_{16} \mathrm{H}_{22} \mathrm{~N}_{2} \mathrm{O}_{4}$ ): 306.15880 (306.15796); MS: $m / z$ (relative intensity) = 306 $\left(\mathrm{M}^{+}, 5\right), 233$ (5), 178 (100), 121 (32), 106 (41); ${ }^{1} \mathrm{H}$ NMR $\left(500 \mathrm{~Hz}, \mathrm{CDCl}_{3}\right): \delta=1.16-1.18(\mathrm{~d}, J=$ $7.00 \mathrm{~Hz}, 3 \mathrm{H}), 1.88(\mathrm{~s}, 3 \mathrm{H}), 2.62-2.66(\mathrm{~m}, 1 \mathrm{H}), 2.81-2.90(\mathrm{~m}, 2 \mathrm{H}), 3.20-3.26(\mathrm{~m}, 1 \mathrm{H}), 3.44-3.48(\mathrm{~m}$, $1 \mathrm{H}), 3.63(\mathrm{~s}, 3 \mathrm{H}), 5.39-5.43(\mathrm{q}, J=6.50 \mathrm{~Hz}, 1 \mathrm{H}), 6.17(\mathrm{br} \mathrm{s}, 1 \mathrm{H}, \mathrm{NH}), 6.86(\mathrm{~d}, J=8.00,1 \mathrm{H}, \mathrm{NH})$, 7.27-7.29 (m, 3H, aroma.), 7.32-7.35 (m, $2 \mathrm{H}$, aroma.); ${ }^{13} \mathrm{C} \mathrm{NMR}\left(126 \mathrm{~Hz}, \mathrm{CDCl}_{3}\right): \delta=15.60$, 23.13, 39.98, 40.62, 42.45, 49.65, 51.93, 126.14, 127.80, 128.83, 140.63, 170.58, 171.55, 174.55; Elemental analysis for $\mathrm{C}_{10} \mathrm{H}_{18} \mathrm{~N}_{2} \mathrm{O}_{4}$ : found $\mathrm{C}, 62.39 \% ; \mathrm{H}, 7.45 \% ; \mathrm{N}, 8.77 \%$, calcd. $\mathrm{C}, 62.73 \%$; $\mathrm{H}$, $7.24 \% ; 9.14 \%$.

$(R, S)-\mathbf{4 f}: \quad$ white solid, m.p $139-140{ }^{\circ} \mathrm{C},{ }^{1} \mathrm{H}$ NMR $\left(500 \mathrm{~Hz}, \mathrm{CDCl}_{3}\right): \delta=1.12-1.13(\mathrm{~d}, J=7.00 \mathrm{~Hz}$, $3 \mathrm{H}), 2.00(\mathrm{~s}, 3 \mathrm{H}), 2.54-2.60(\mathrm{~m}, 1 \mathrm{H}), 2.83-2.84(\mathrm{~d}, J=6.50 \mathrm{~Hz}, 2 \mathrm{H}), 3.17-3.22(\mathrm{~m}, 1 \mathrm{H}), 3.50-3.54$ $(\mathrm{m}, 1 \mathrm{H}), 3.66(\mathrm{~s}, 3 \mathrm{H}), 5.40-5.44(\mathrm{q}, J=6.50 \mathrm{~Hz}, 1 \mathrm{H}), 6.63(\mathrm{br} \mathrm{s}, 1 \mathrm{H}, \mathrm{NH}), 6.95-6.97(\mathrm{~d}, J=8.00$ $\mathrm{Hz}, 1 \mathrm{H}, \mathrm{NH}), 7.26-7.29$ (m, 3H, aroma.), 7.32-7.35 (m, 2H, aroma.); ${ }^{13} \mathrm{C} \mathrm{NMR}\left(126 \mathrm{~Hz}, \mathrm{CDCl}_{3}\right): \delta=$ $15.43,23.14,40.42,40.74,42.58,49.86,52.03,126.18,127.83,128.88,140.49,170.79,171.94$, 174.51 .

Ethyl 3-(3-N-acetylaminobutanoyl)aminopropanoate 4g.

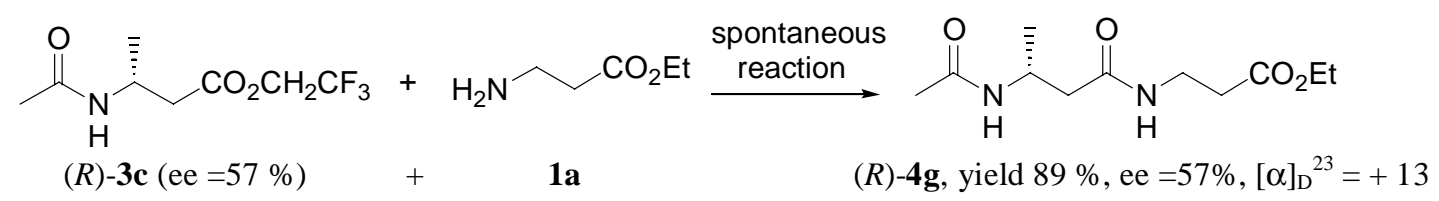

$(R)-\mathbf{3 c}(0.18 \mathrm{mmol}, 41 \mathrm{mg})$ and $\mathbf{1 a}(0.35 \mathrm{mmol}, 41 \mathrm{mg})$ were let to react in dry diethyl ether $(1.76$ $\mathrm{mL})$. Following the above procedure $(R)-4 \mathrm{~g}\left(0.16 \mathrm{mmol}, 39 \mathrm{mg}\right.$, ee $\left.=57 \%,[\alpha]_{\mathrm{D}}=+13\right)$. was obtained at $89 \%$ isolated yield, white solid, m.p. $119-120{ }^{\circ} \mathrm{C} ; \mathrm{HRMS} \mathrm{M}^{+}$found $\left(\mathrm{M}^{+}\right.$calculated for $\mathrm{C}_{11} \mathrm{H}_{20} \mathrm{~N}_{2} \mathrm{O}_{4}$ ): 244.14230 (244.14231); $\mathrm{MS}: m / z$ (relative intensity) = $244\left(\mathrm{M}^{+}, 17\right), 201(25), 140$ (23), 116 (30), 84 (40), $44(100)$; ${ }^{1} \mathrm{H}$ NMR $\left(500 \mathrm{~Hz}, \mathrm{CDCl}_{3}\right): \delta=1.21(\mathrm{~d}, J=7.00,3 \mathrm{H}), 1.28(\mathrm{t}, J=$ $7.00 \mathrm{~Hz}, 3 \mathrm{H}), 1.97(\mathrm{~s}, 3 \mathrm{H}), 2.29-2.33(\mathrm{dd}, J=14.50,5.00 \mathrm{~Hz}, 1 \mathrm{H}), 2.45-2.49$ (dd, $J=14.50,5.00$ $\mathrm{Hz}, 1 \mathrm{H}), 2.54(\mathrm{t}, J=6.00 \mathrm{~Hz}, 2 \mathrm{H}), 3.48-3.56(\mathrm{~m}, 2 \mathrm{H}), 4.14-4.18(\mathrm{q}, J=7.50 \mathrm{~Hz}, 2 \mathrm{H}), 4.27-4.29(\mathrm{~m}$, $1 \mathrm{H}), 6.53$ (br s, $1 \mathrm{H}, \mathrm{NH}), 6.80($ br s, $1 \mathrm{H}, \mathrm{NH}) ;{ }^{13} \mathrm{C} \mathrm{NMR}\left(126 \mathrm{~Hz}, \mathrm{CDCl}_{3}\right): \delta=14.18,19.97,23.41$, 33.94, 34.92, 41.47, 42.75, 60.86, 170.28, 171.22, 172.48; Elemental analysis for $\mathrm{C}_{11} \mathrm{H}_{20} \mathrm{~N}_{2} \mathrm{O}_{4}$ : found $\mathrm{C}, 54.27 \% ; \mathrm{H}, 8.33 \% ; 11.27 \%$, calcd. C, $54.08 \% ; \mathrm{H}, 8.25 \% ; \mathrm{N}, 11.47 \%$.

\section{References}

(1) Solymár, M.; Liljeblad, A.; Lázár, L.; Fülöp, F.; Kanerva, L. T. Tetrahedron: Asymmetry 2002, $13,1923$.

(2) Gedey, S.; Liljeblad, A.; Fülöp, F.; Kanerva, L. T. Tetrahedron: Asymmetry 1999, 10, 2573. 
(3) Gedey, S.; Liljeblad, A.; Lázár, L.; Fülöp, F.; Kanerva, L. T. Tetrahedron: Asymmetry 2001, 12, 105.

(4) Kanerva, L. T.; Sundholm, O. J. Chem. Soc., Perkin Trans. 1 1993, 2407.

\section{Copies of NMR spectra}



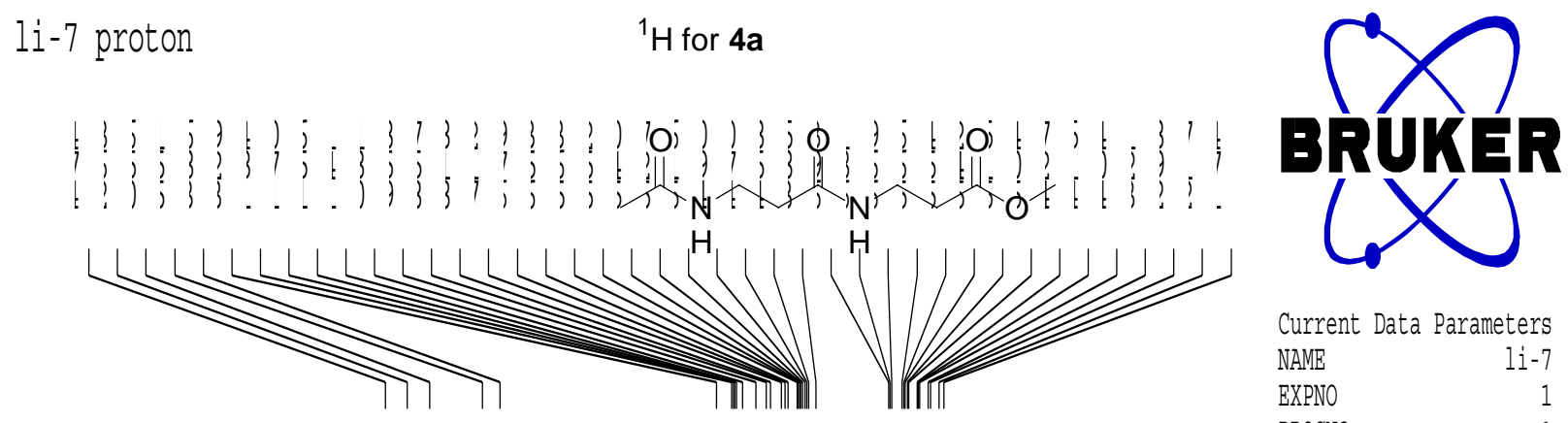

Current Data Parameters

NAME

EXPNO

PROCNO

li-7

F2 - Acquisition Parameters

Date 20060703

Time $\quad 12.32$

INSTRUM AV500

PROBHD $5 \mathrm{~mm}$ PABBI 1H-

PULPROG 2930

TD 65536

SOLVENT $\quad \mathrm{CDCl3}$

NS 16

DS 2

SWH $\quad 10330.578 \mathrm{~Hz}$

FIDRES $\quad 0.157632 \mathrm{~Hz}$

AO $\quad 3.1720407 \mathrm{sec}$

RG 203.2

DW $\quad 48.400$ usec

DE $\quad 6.00$ usec

TE $\quad 298.1 \mathrm{~K}$

D1 $\quad 1.00000000 \mathrm{sec}$

MCREST $\quad 0.00000000 \mathrm{sec}$

MCWRK $\quad 0.01500000 \mathrm{sec}$

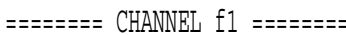

NUC1 $1 \mathrm{H}$

P1 $\quad 6.90$ usec

PL1 $\quad 1.50 \mathrm{~dB}$

SF01 $\quad 500.1330885 \mathrm{MHz}$

F2 - Processing parameters

SI 32768

SF $\quad 500.1300097 \mathrm{MHz}$

WDW EM

SSB 0

LB $\quad 0.30 \mathrm{~Hz}$

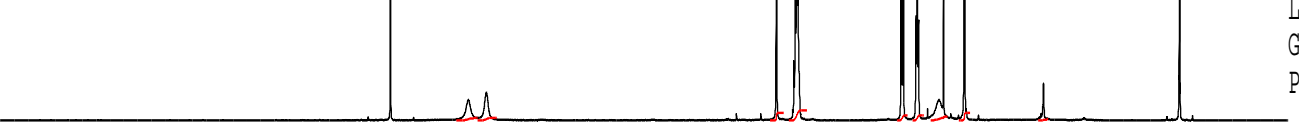

0

PC $\quad 1.00$

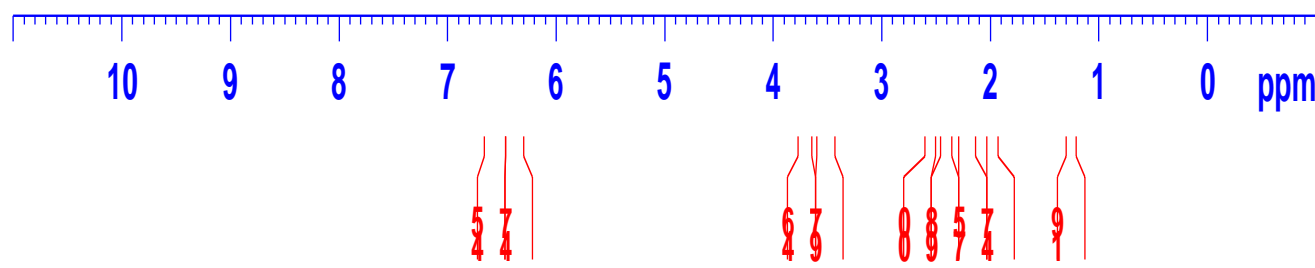



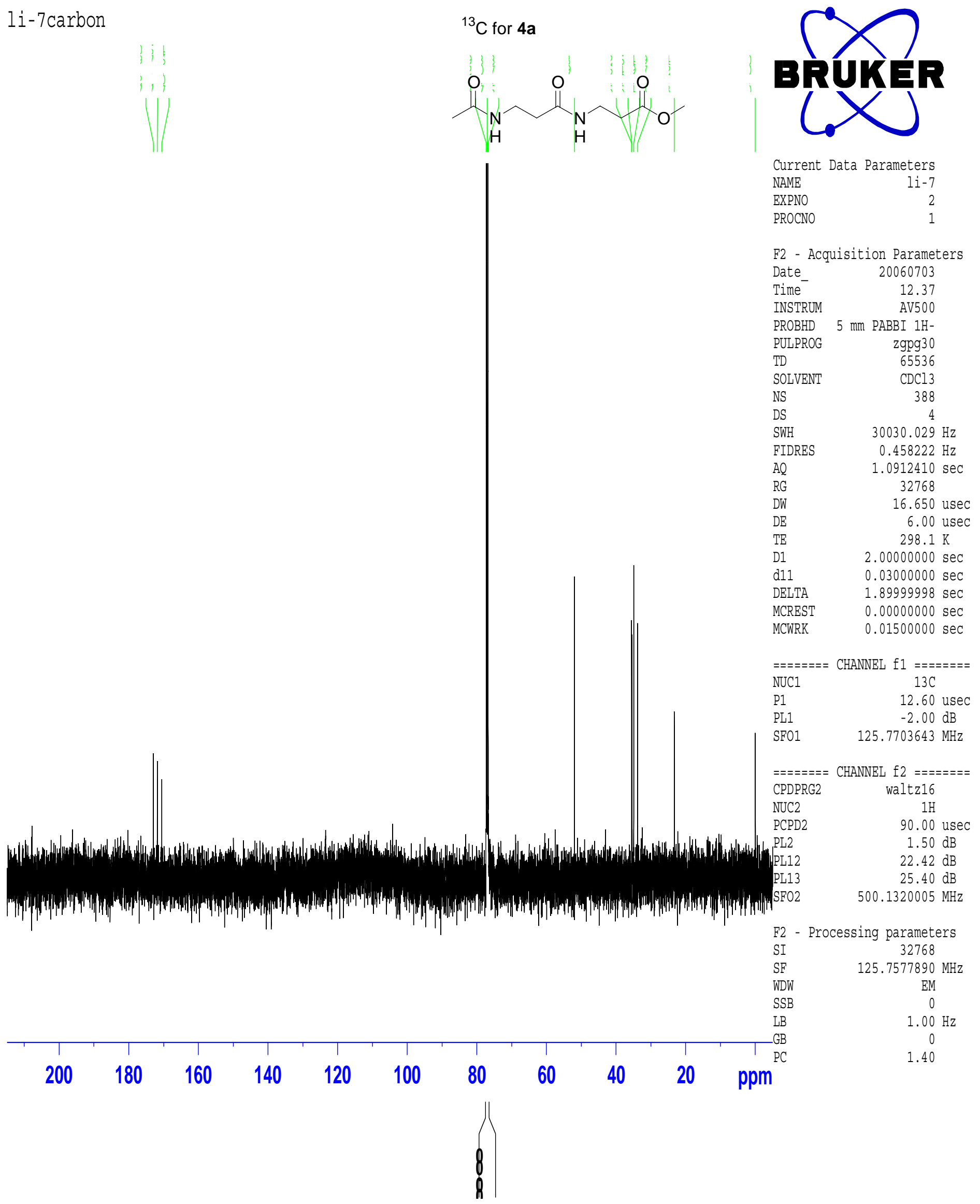


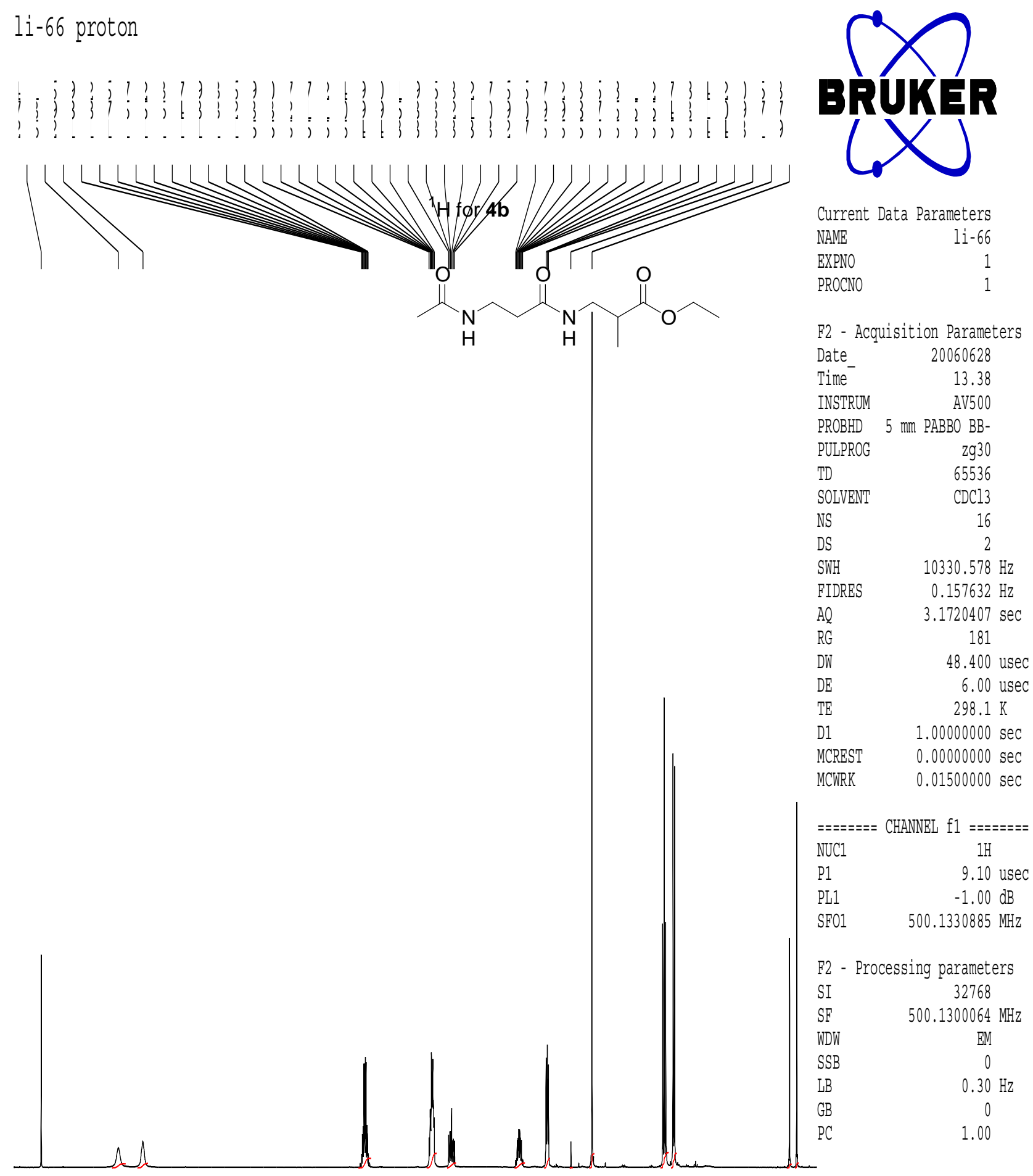

\section{$\begin{array}{lllllllllllllll}7.0 & 6.5 & 6.0 & 5.5 & 5.0 & 4.5 & 4.0 & 3.5 & 3.0 & 2.5 & 2.0 & 1.5 & 1.0 & 0.5 & \mathrm{ppm}\end{array}$ $5|3|$

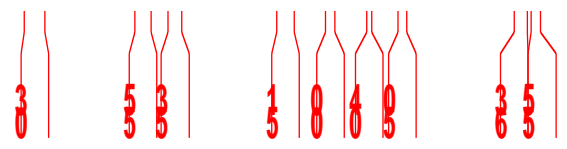 \\ 35}




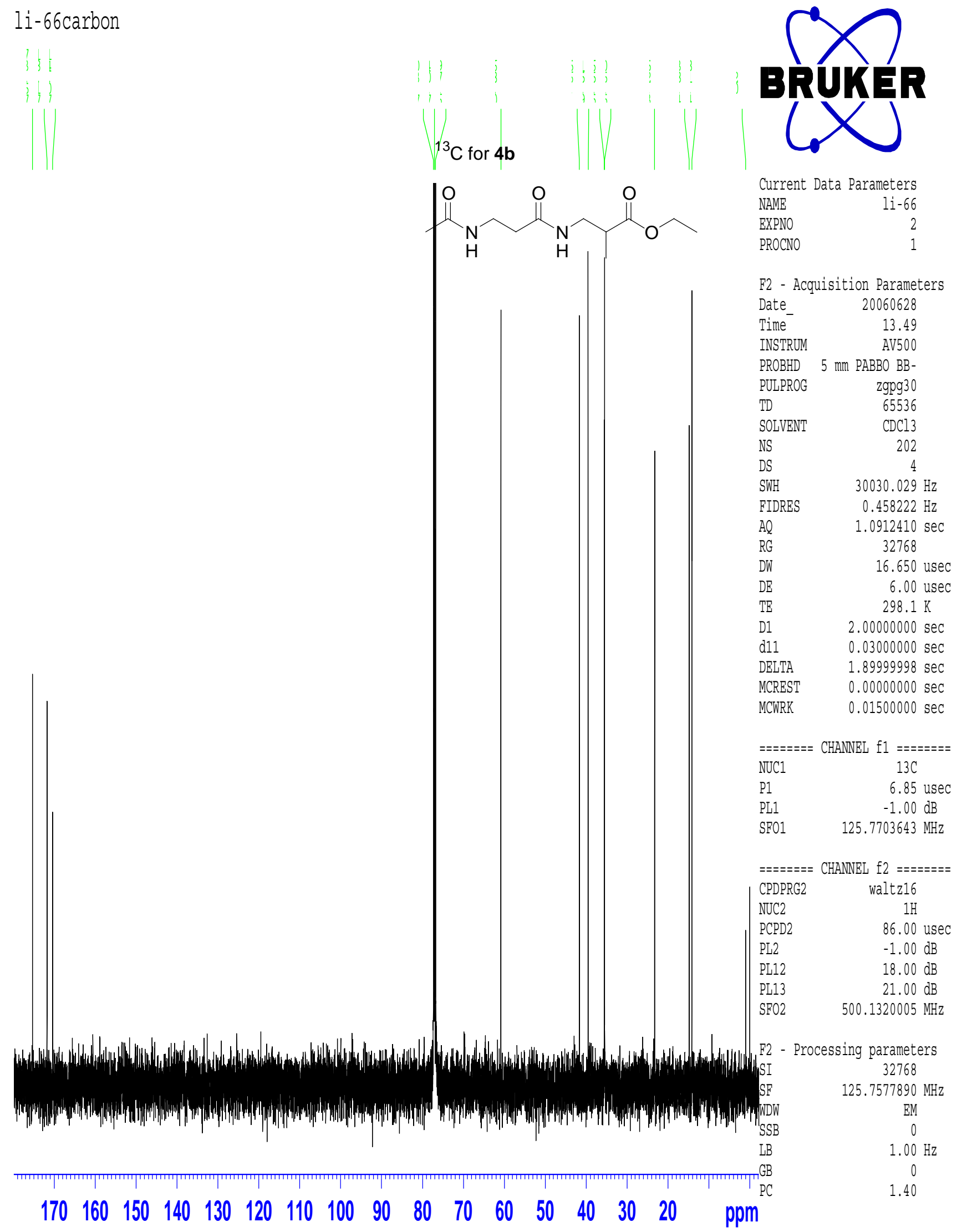




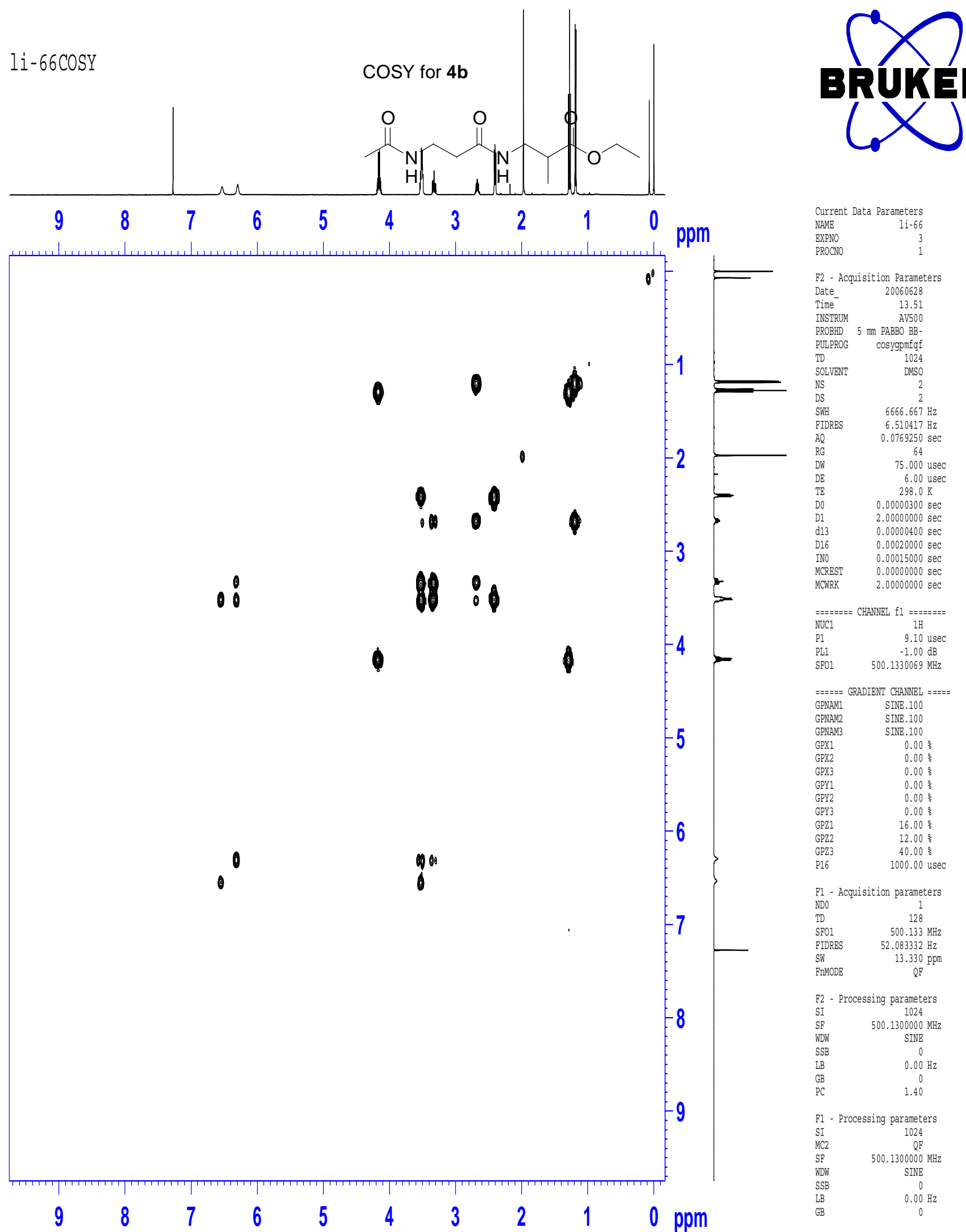




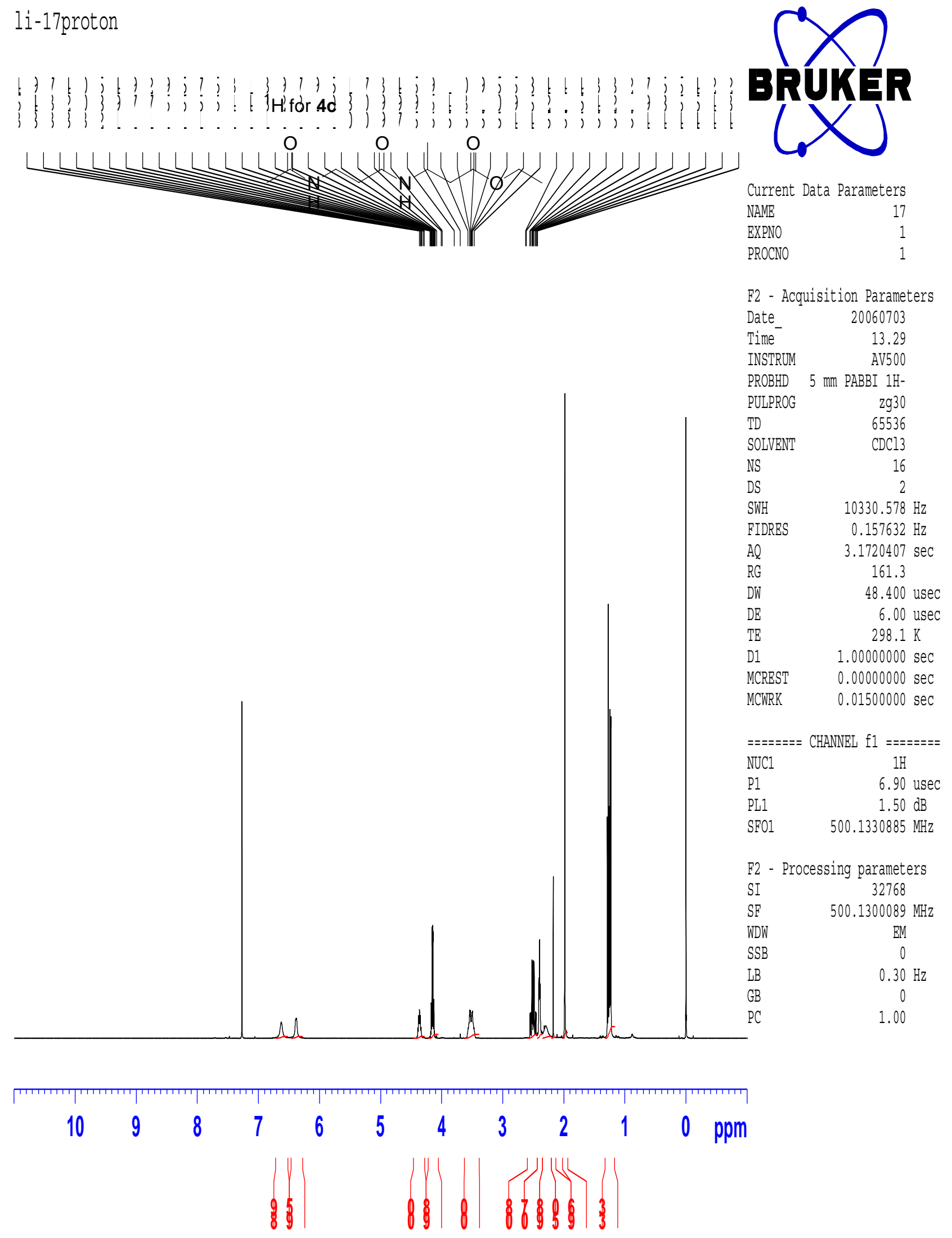




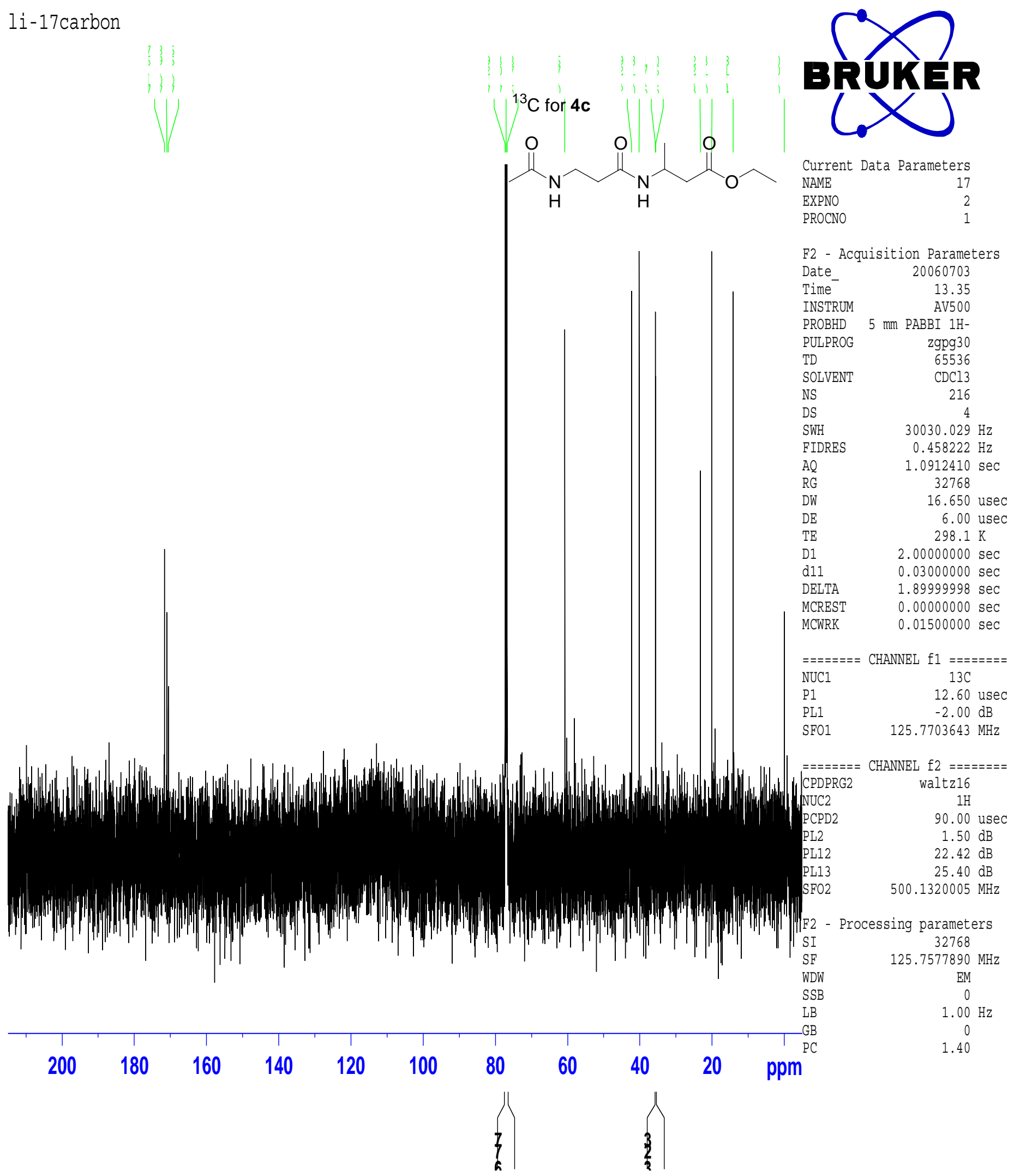




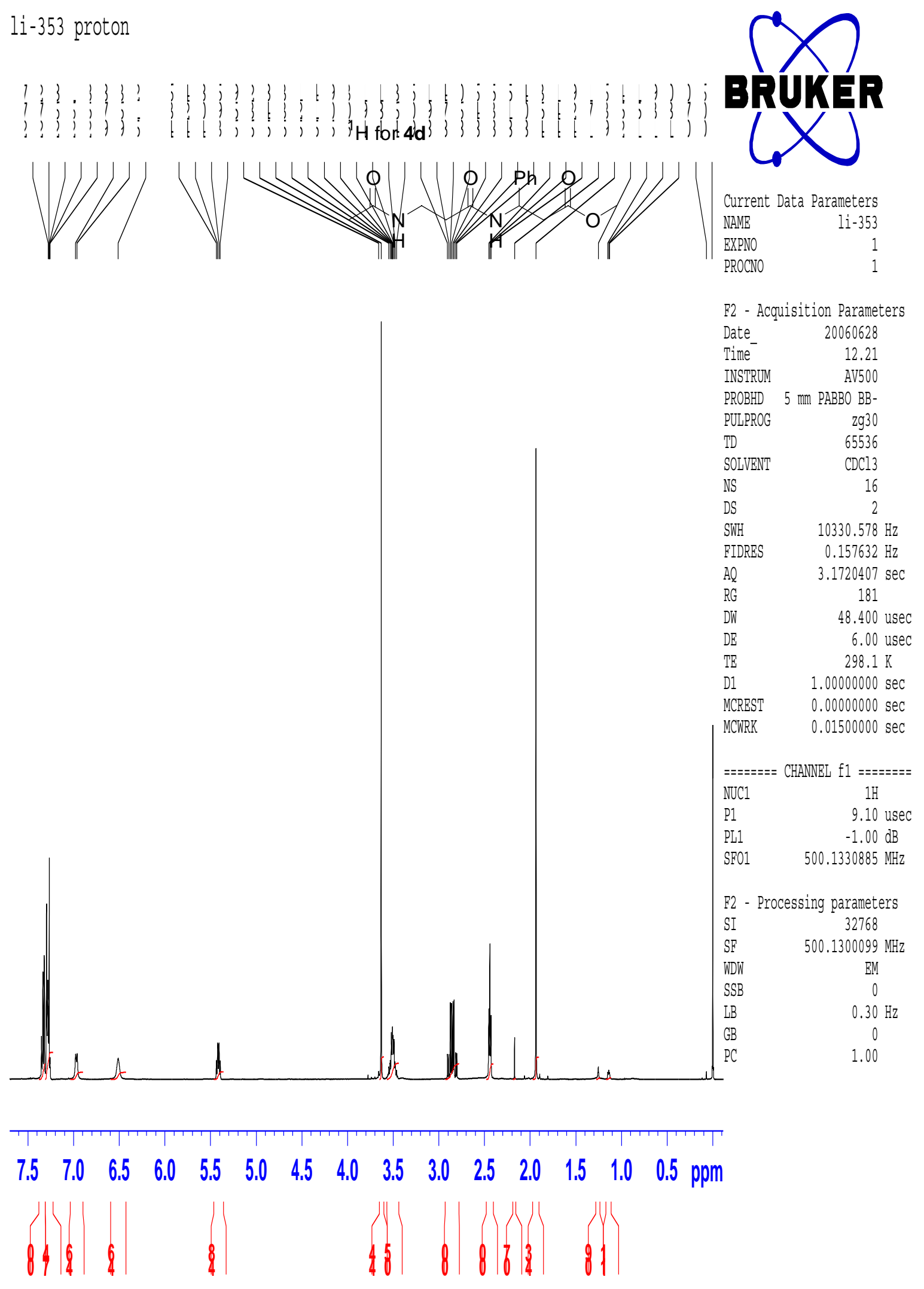




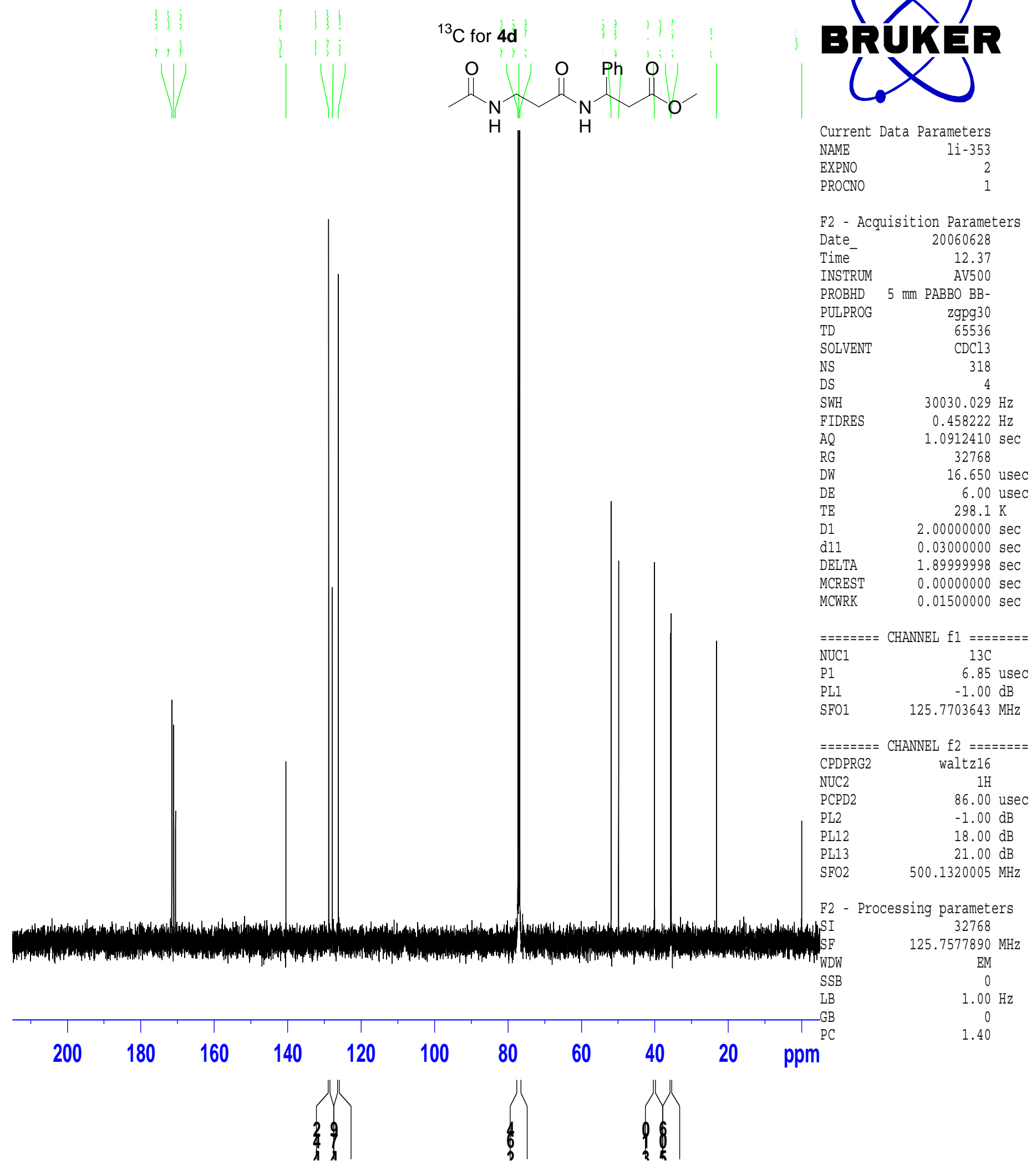




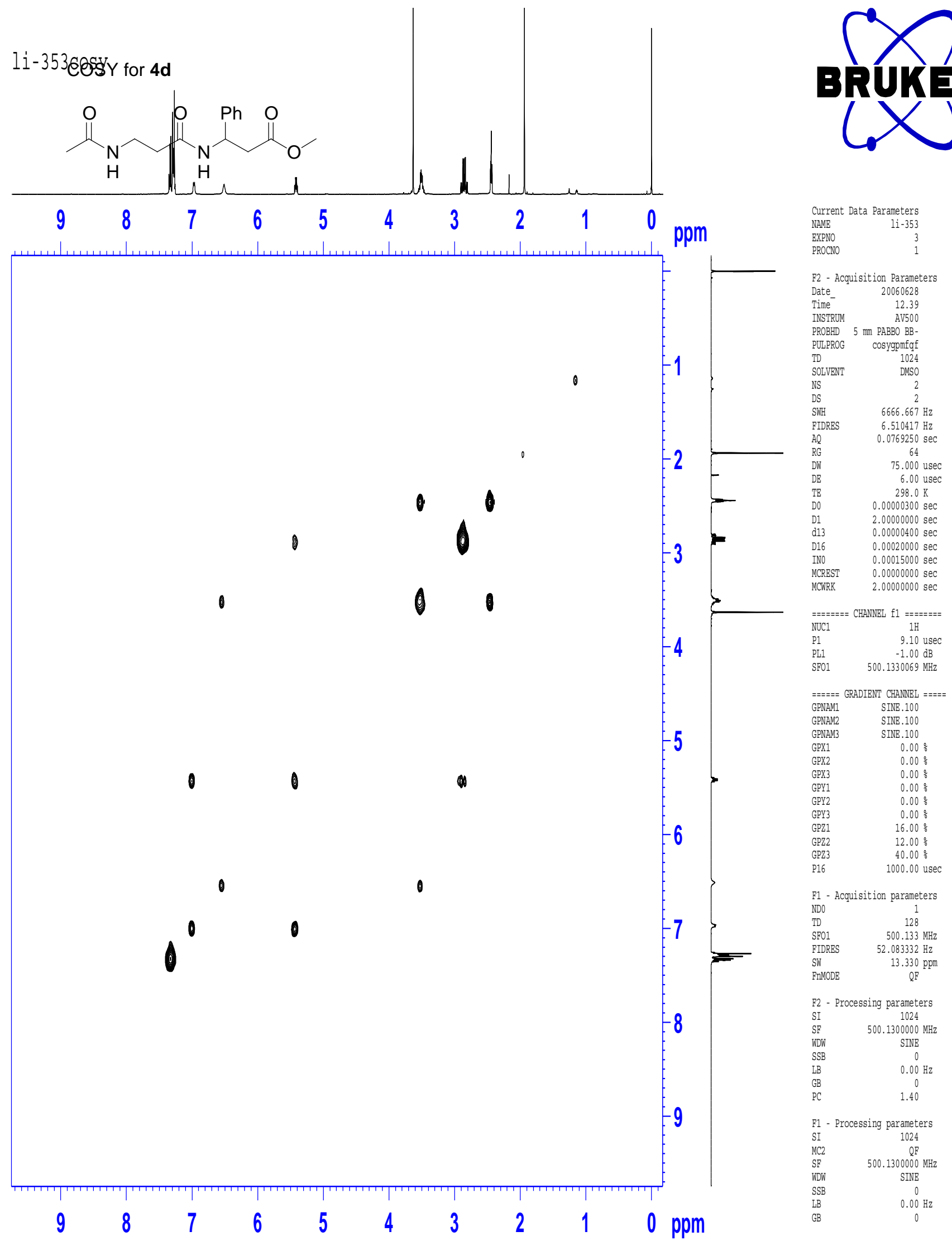




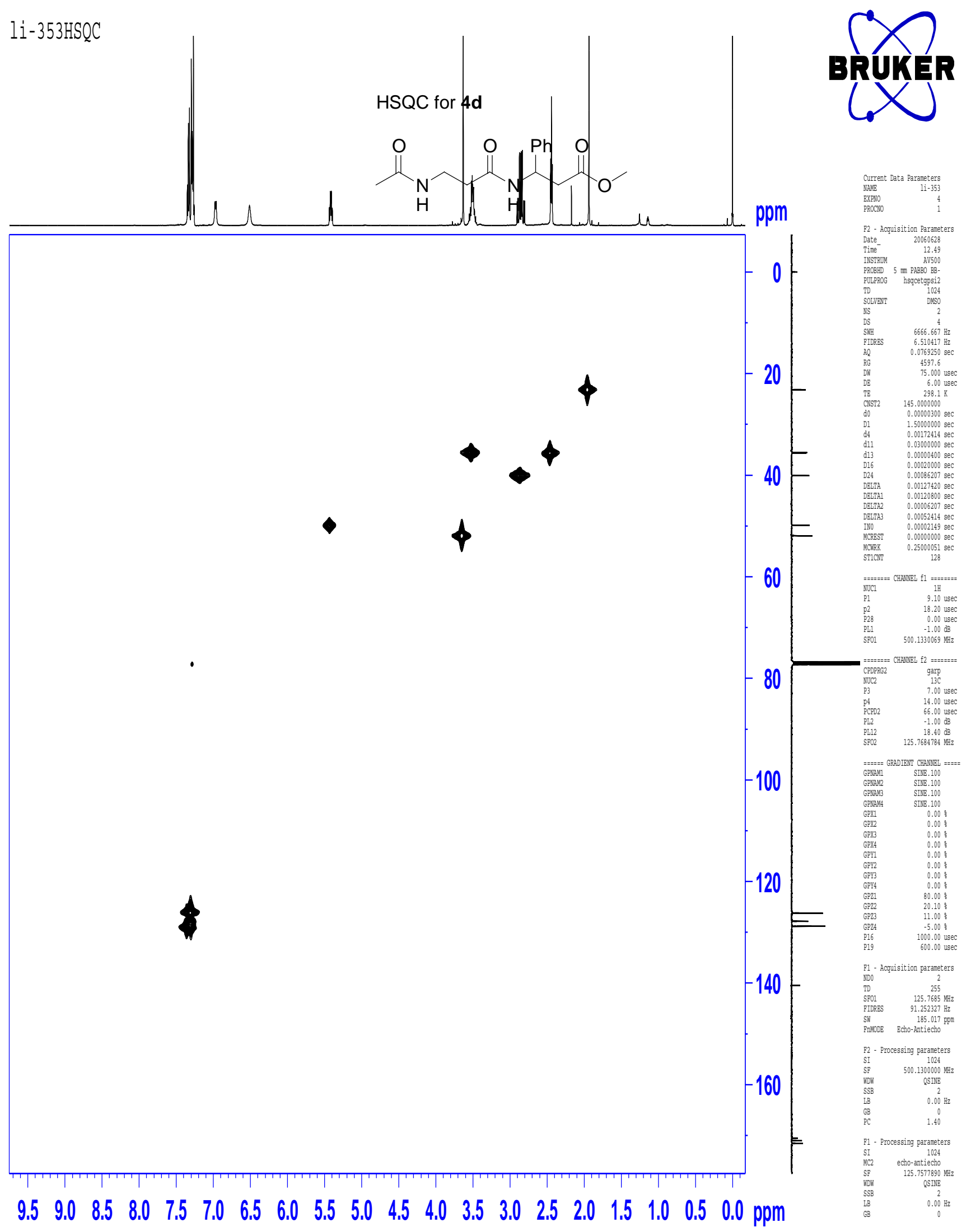




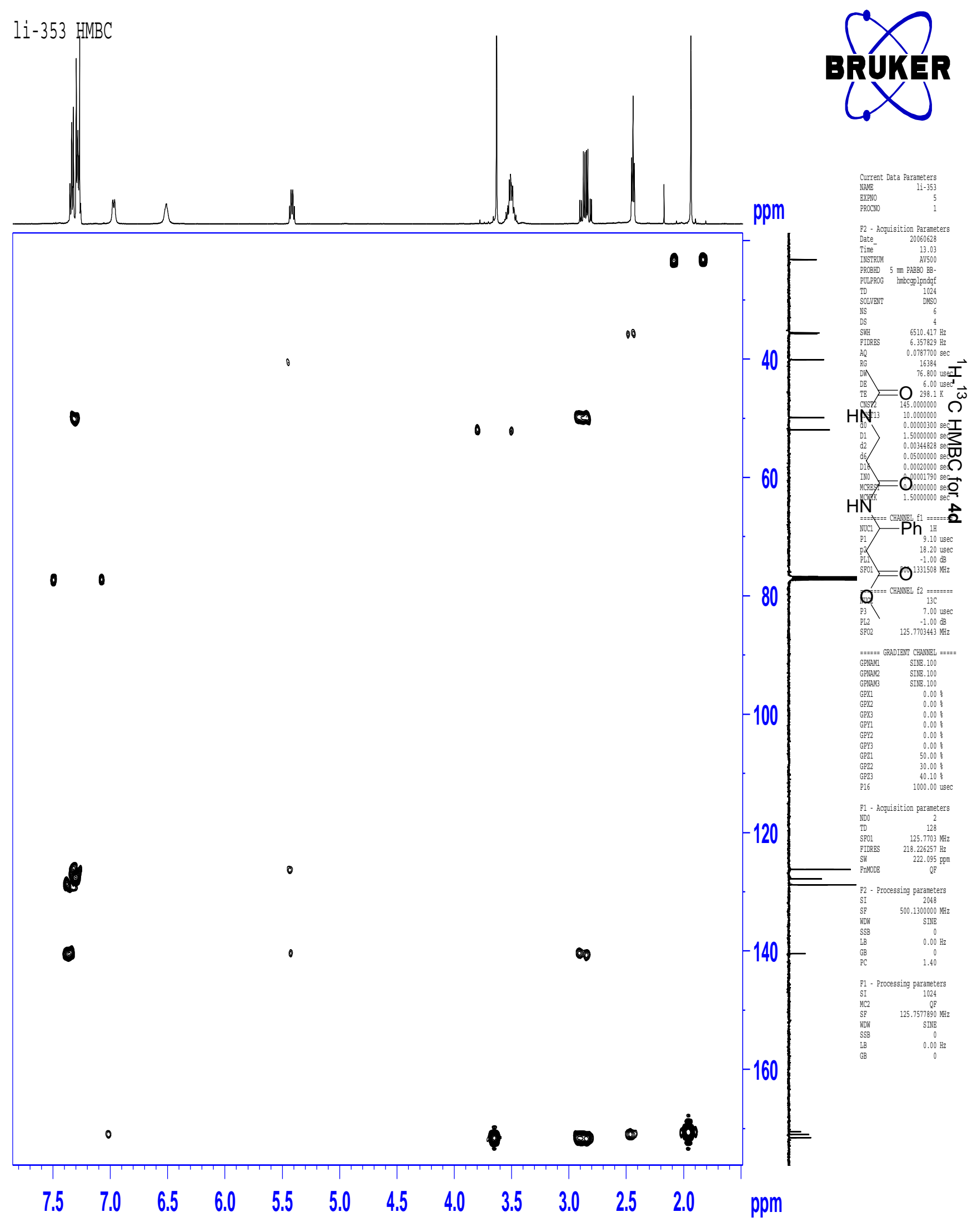


1i-71 proton
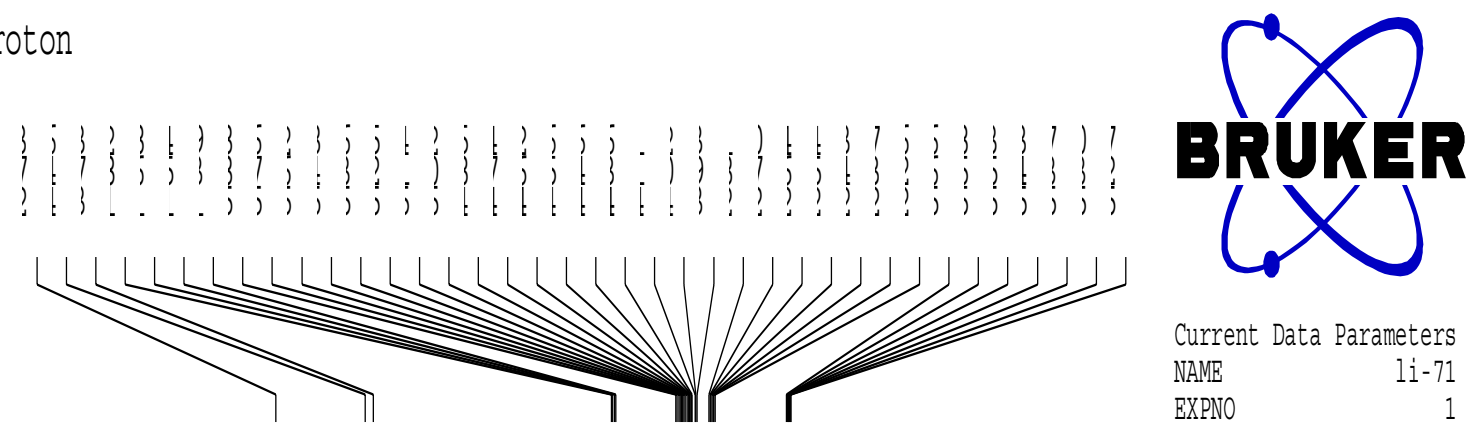

$\begin{array}{lr}\text { Current } & \text { Data Parameters } \\ \text { NAME } & 1 \mathrm{i}-71 \\ \text { EXPNO } & 1 \\ \text { PROCNO } & 1\end{array}$

F2 - Acquisition Parameters
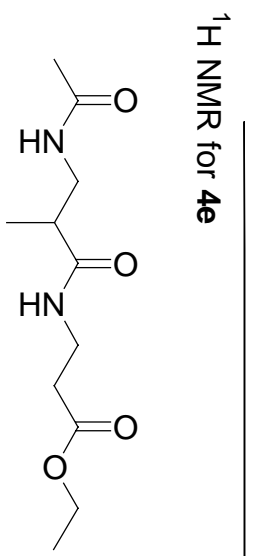

Date

Time $\quad 11.58$

INSTRUMM AV500

PROBHD $5 \mathrm{~mm}$ PABBI $1 \mathrm{H}-$

PULPROG

TD 65536

SOLVENT $\quad$ CDCl3

NS

16

DS

SWH

FIDRES

$\mathrm{AO}$
$\mathrm{RG}$

DW

$D E$

TE

D1

MCREST

MCWRK

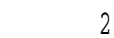

$10330.578 \mathrm{~Hz}$

$0.157632 \mathrm{~Hz}$

$3.1720407 \mathrm{sec}$ 128

48.400 usec 6.00 usec $298.1 \mathrm{~K}$

$1.00000000 \mathrm{sec}$

$0.00000000 \mathrm{sec}$

$0.01500000 \mathrm{sec}$

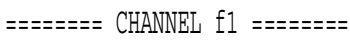
NUC1

P1 $\quad 6.90$ usec

PL1

SF01 $\quad 500.1330885 \mathrm{MHz}$

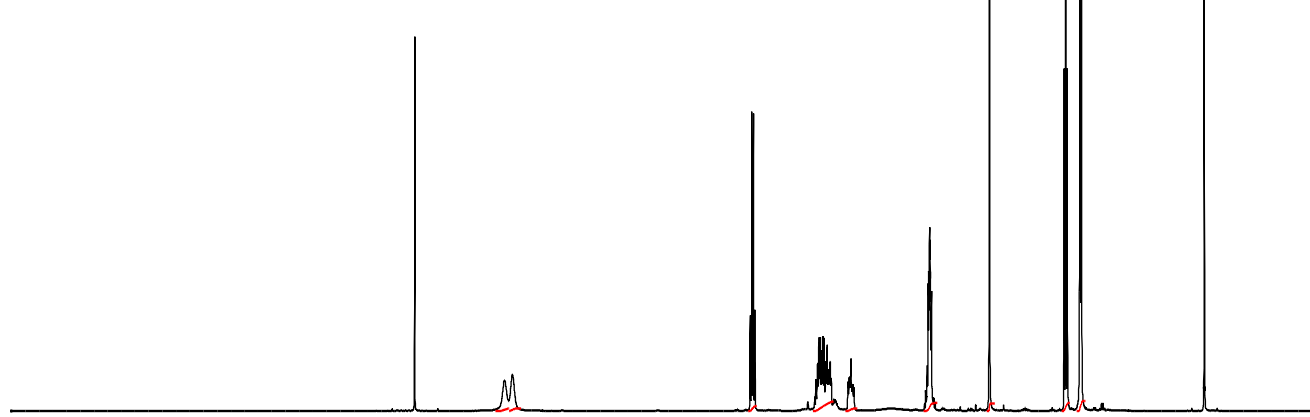

F2 - Processing parameters

SI 32768

SF $\quad 500.1300072 \mathrm{MHz}$

WDW

SSB

LB

$G B$
$P C$

$\mathrm{EM}$
$\mathrm{O}$

$0.30 \mathrm{~Hz}$

0

1.00

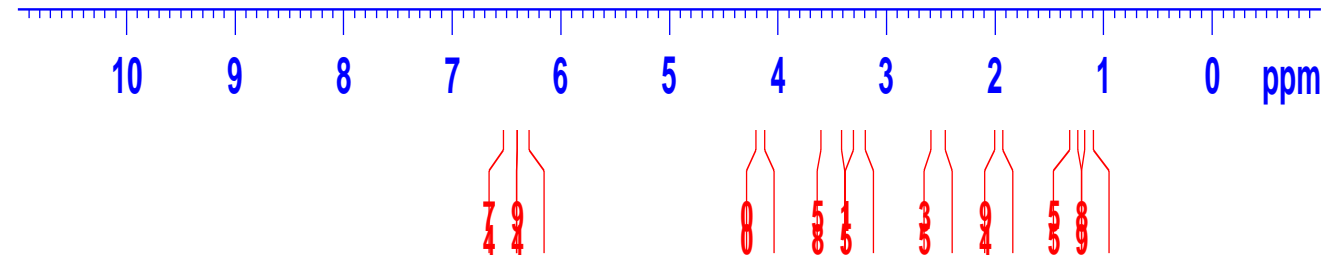




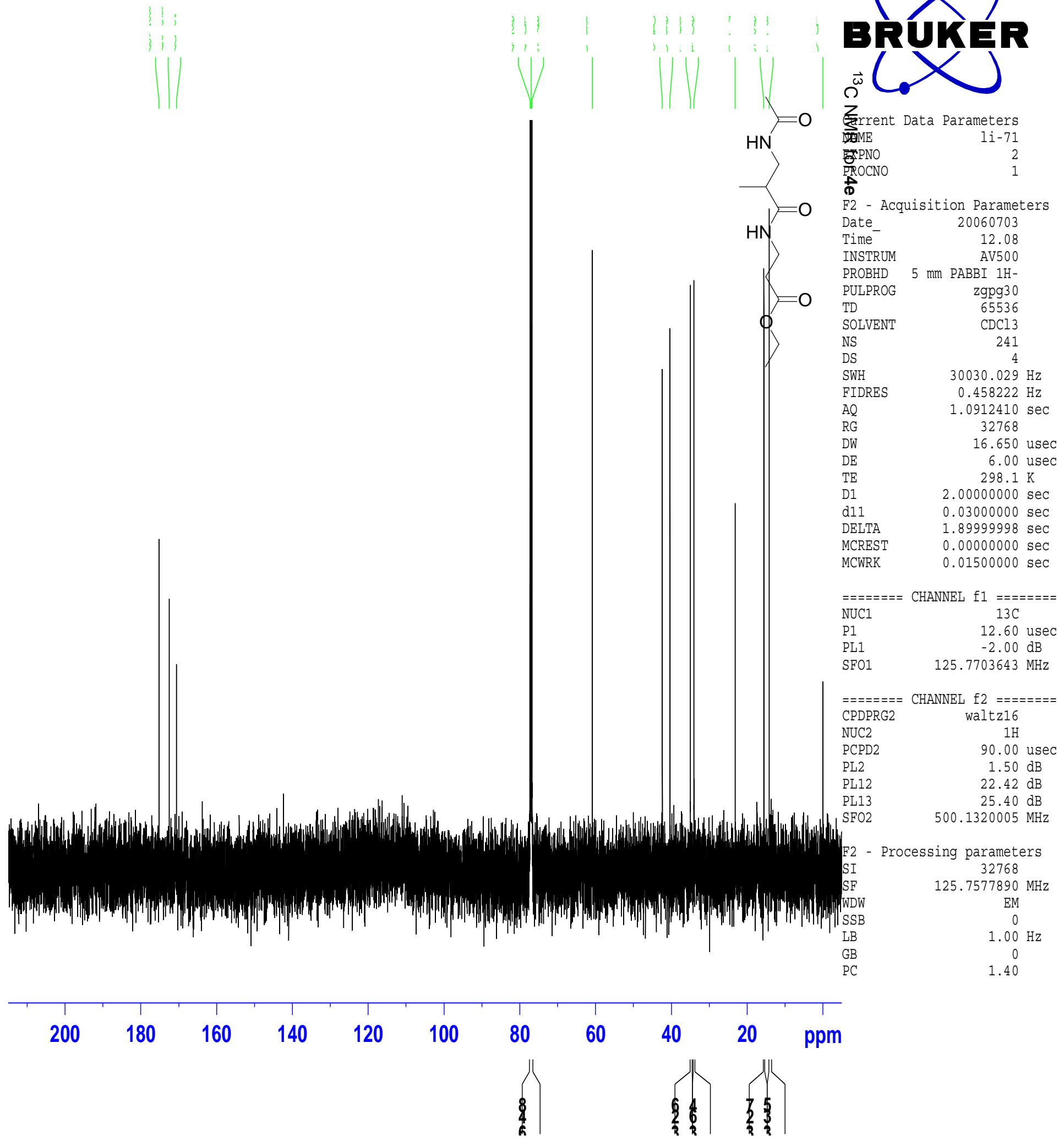




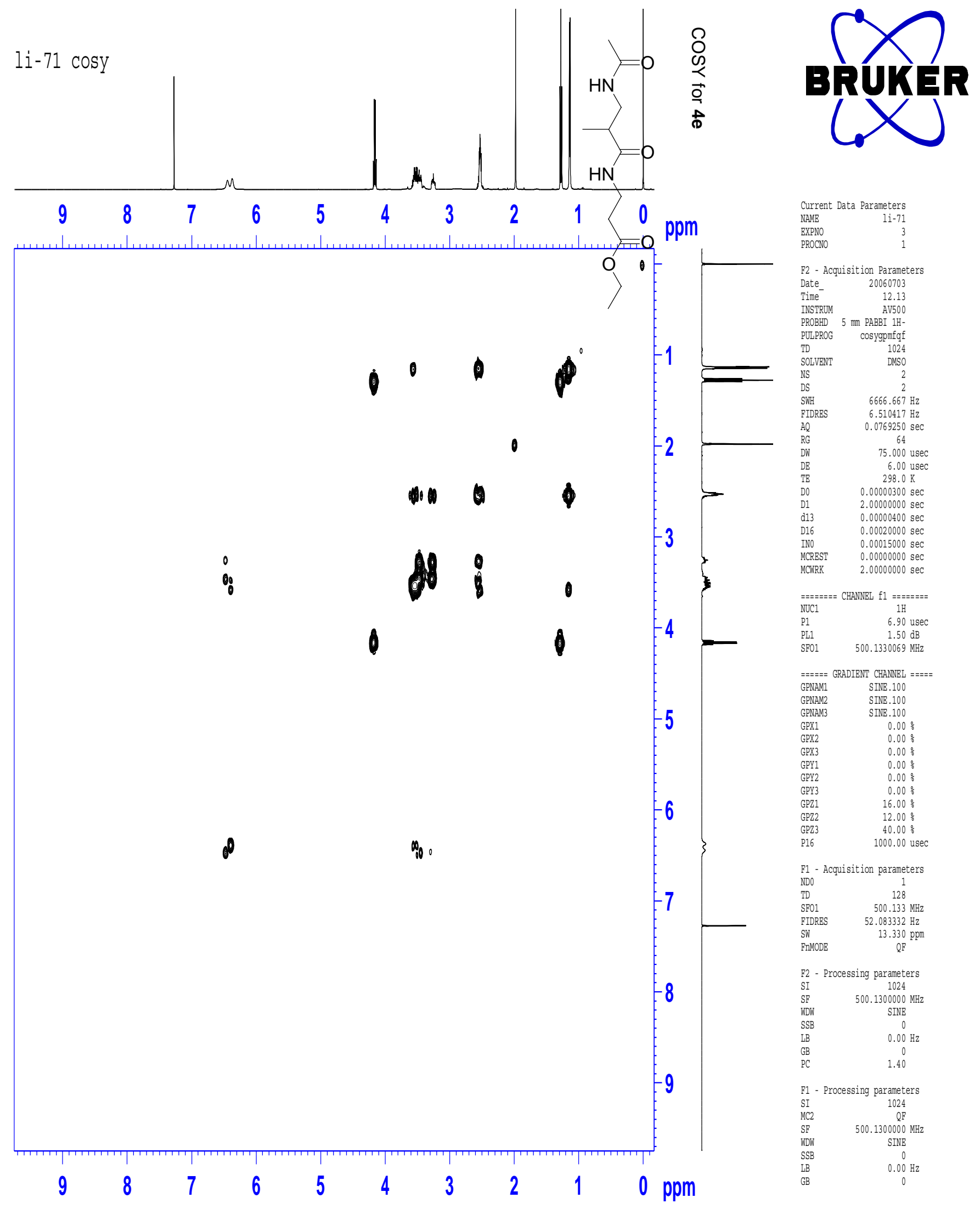




\section{III508SS PROTON}

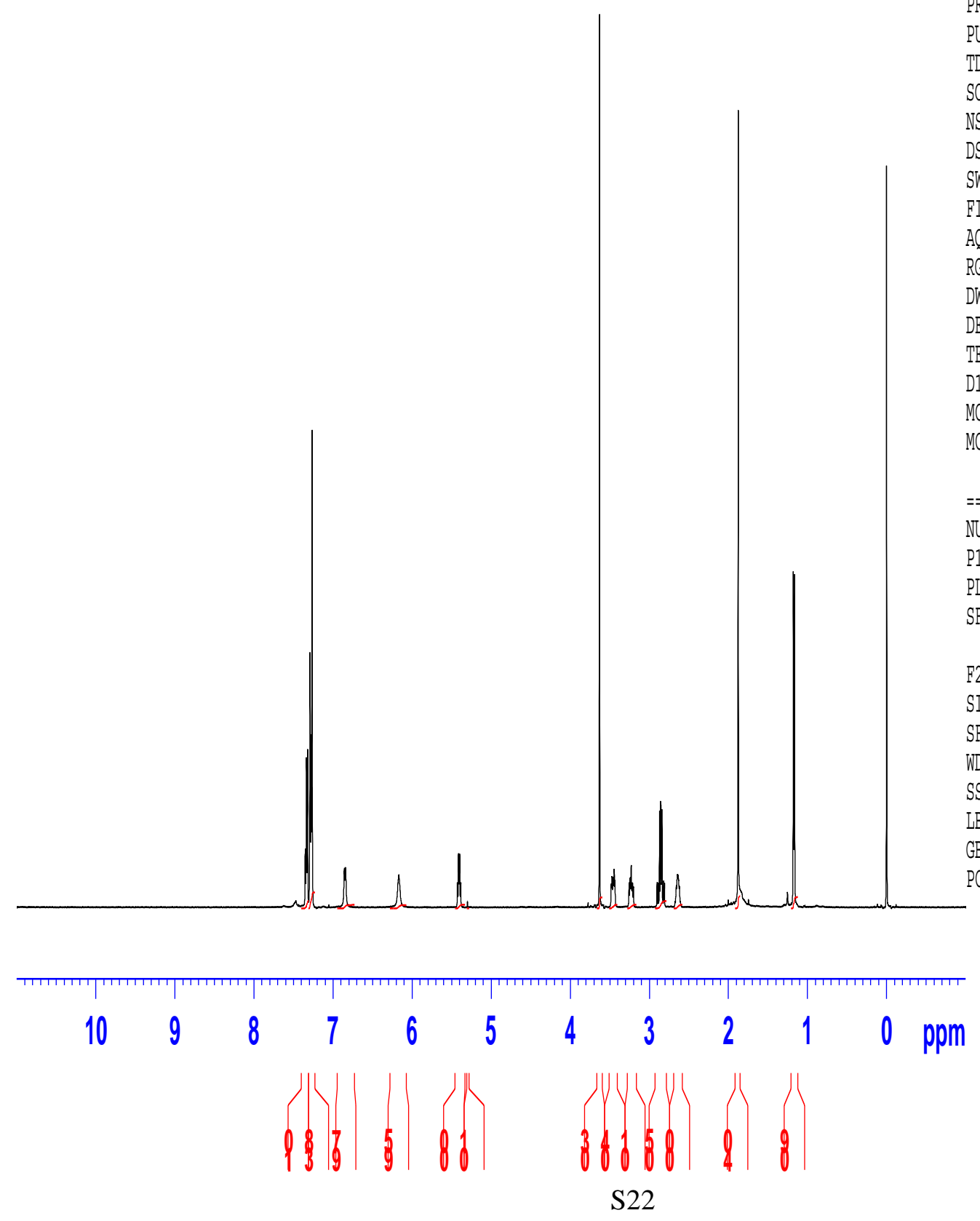




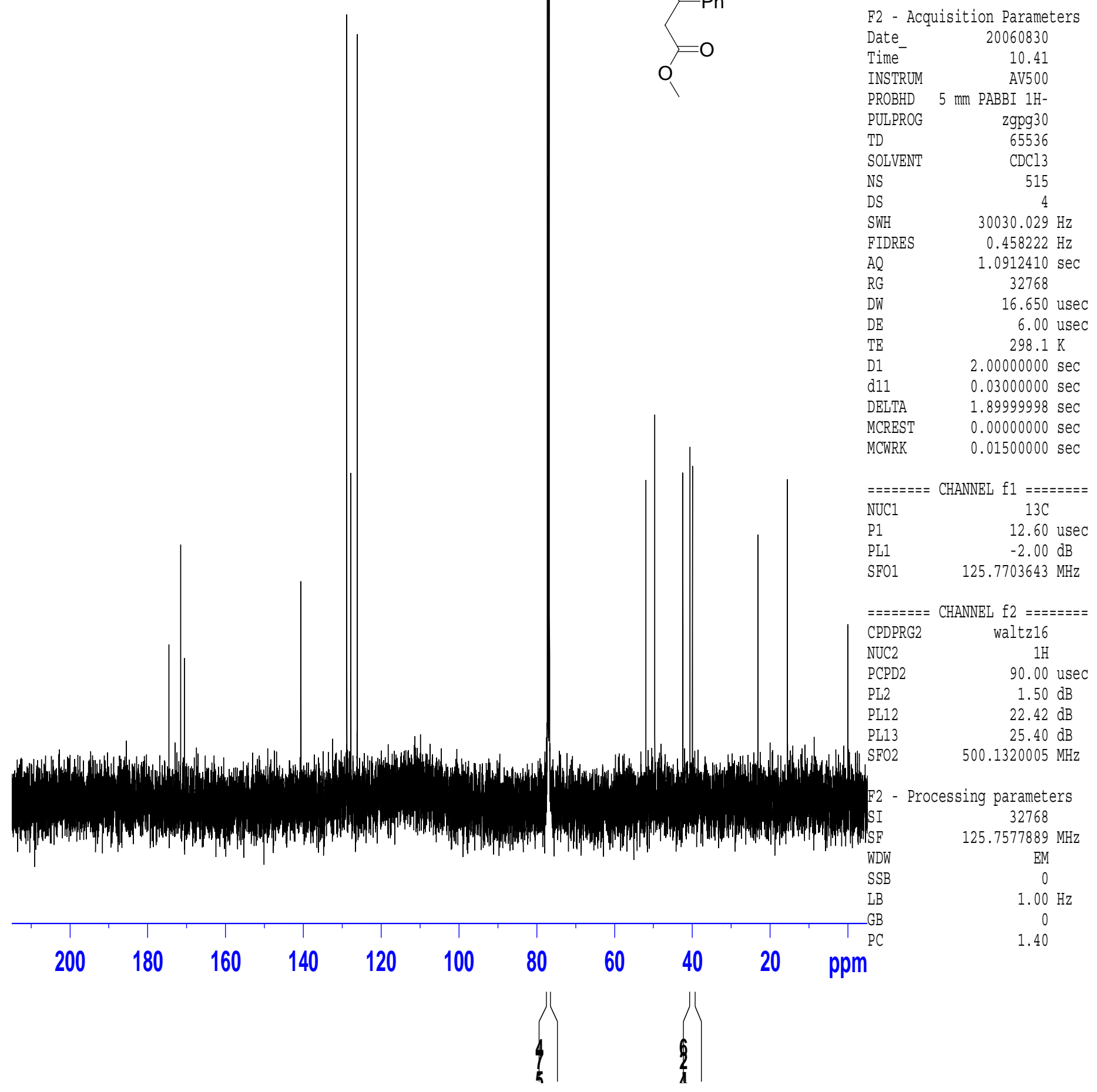



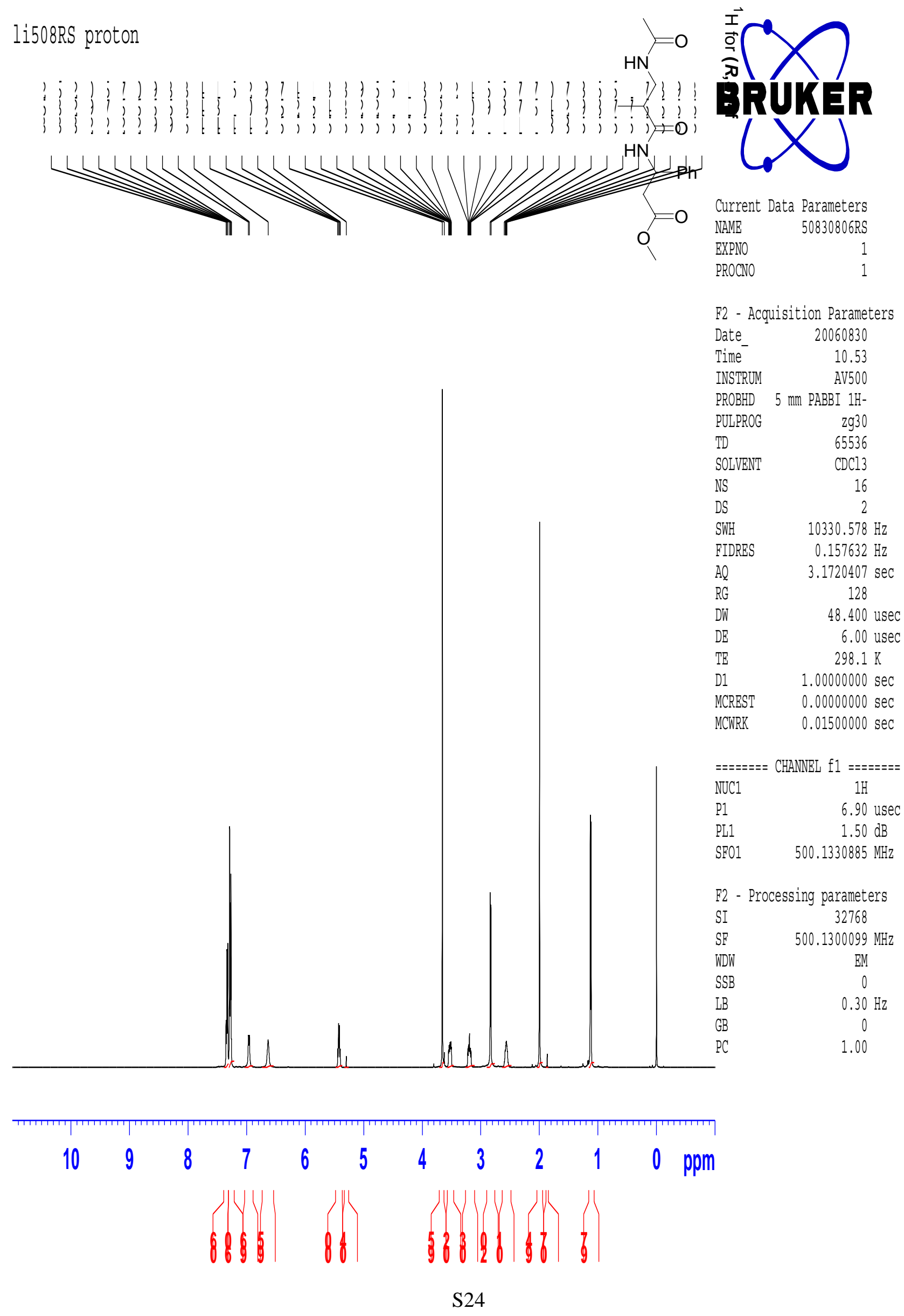


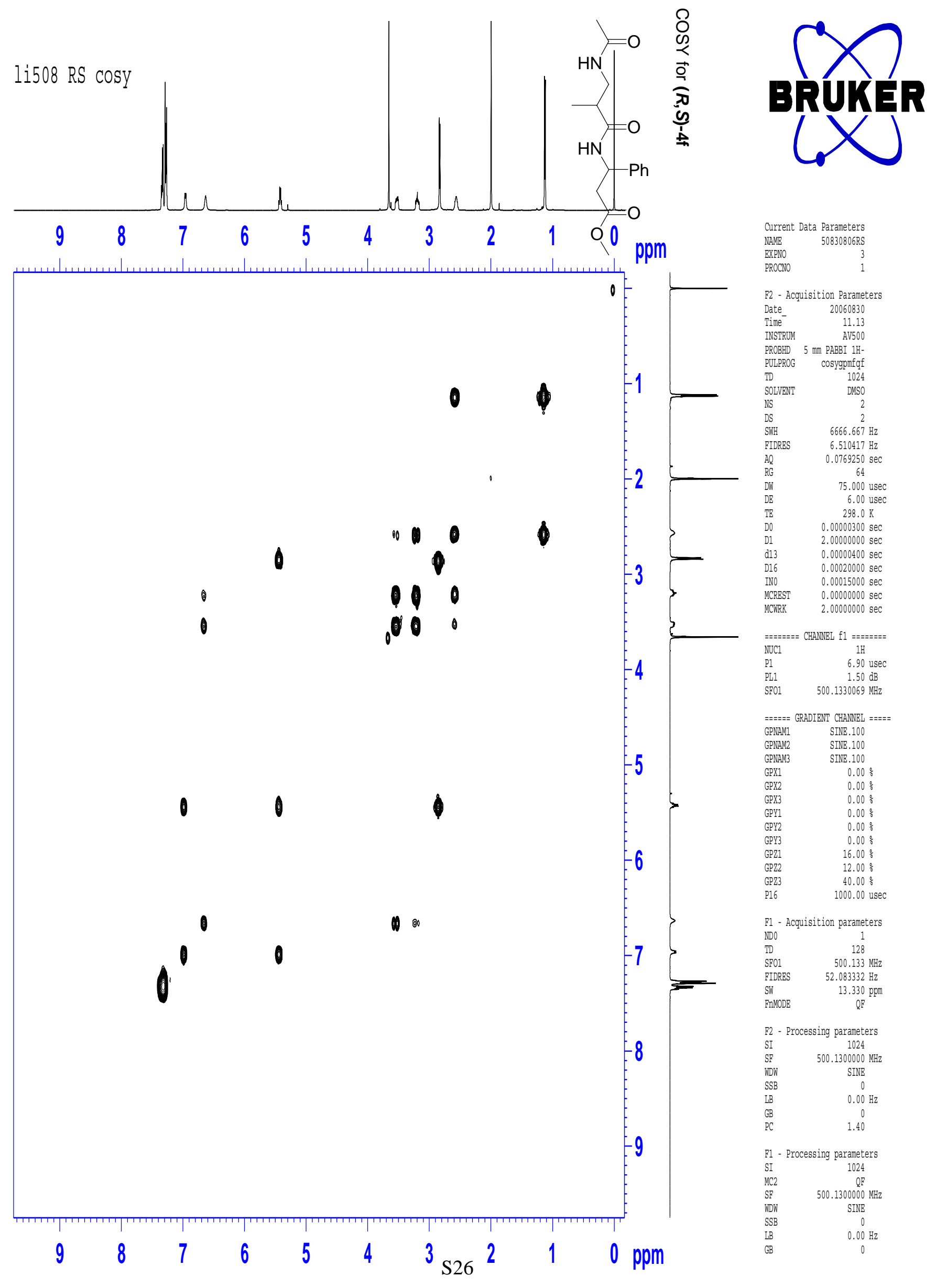




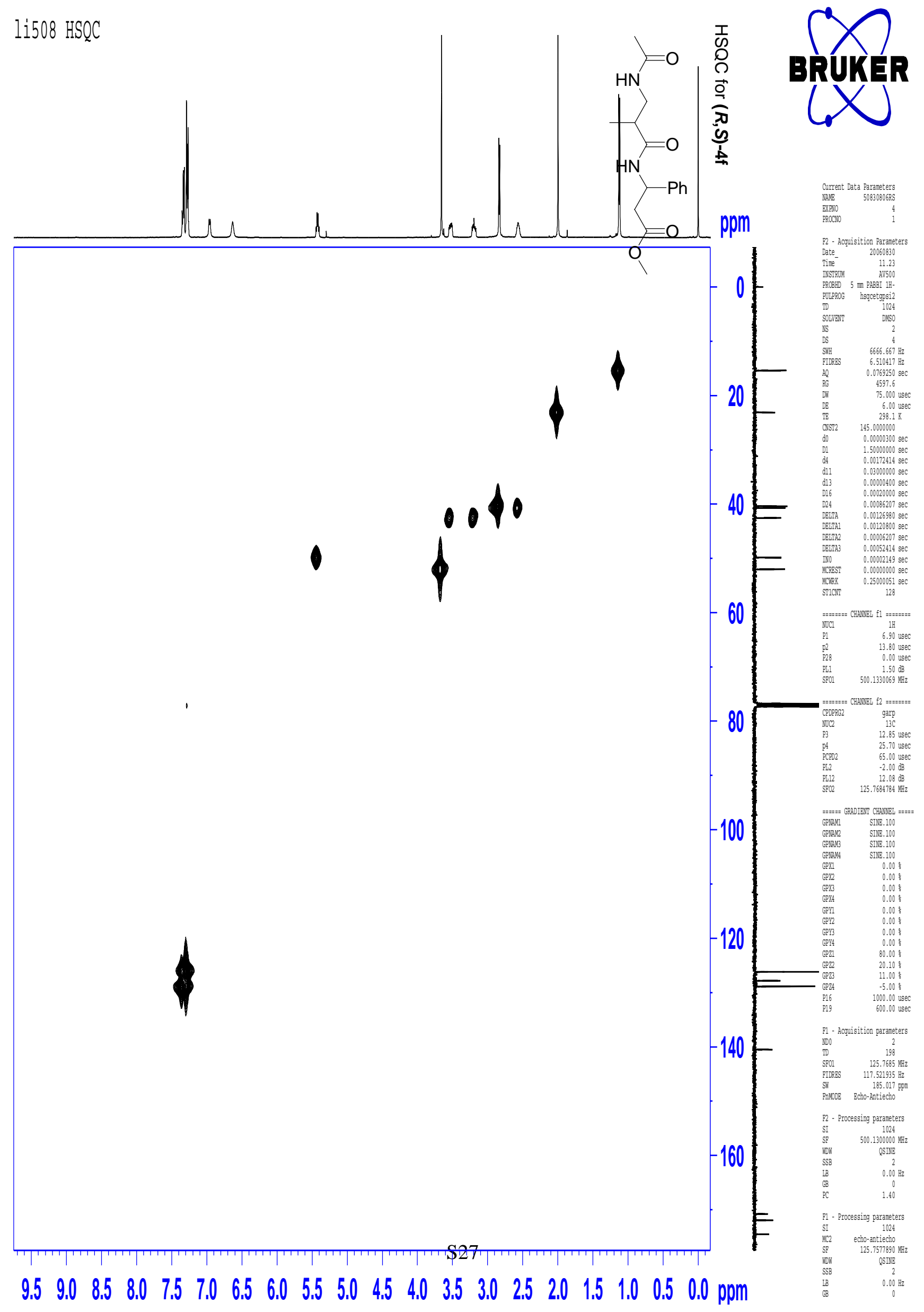




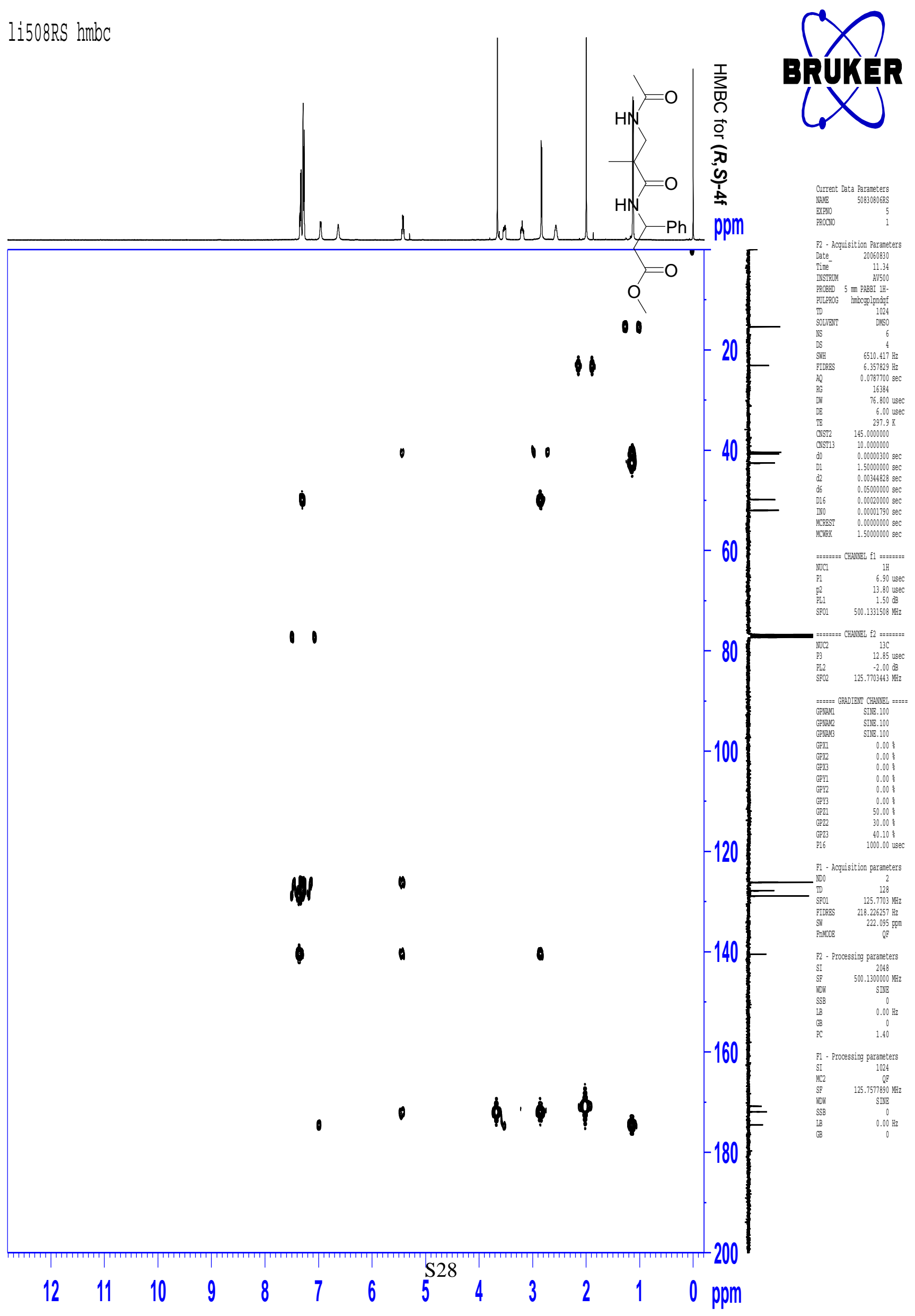




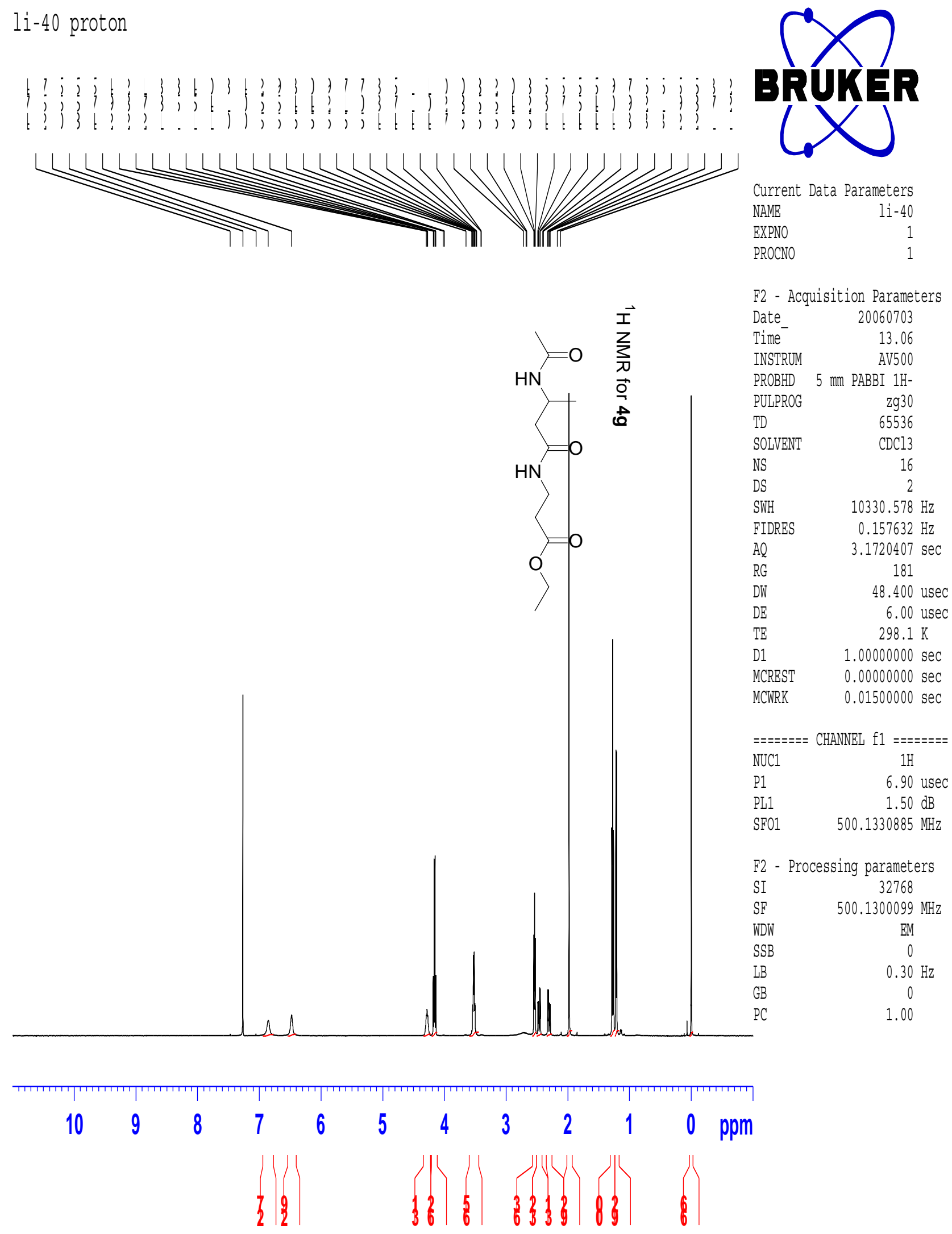



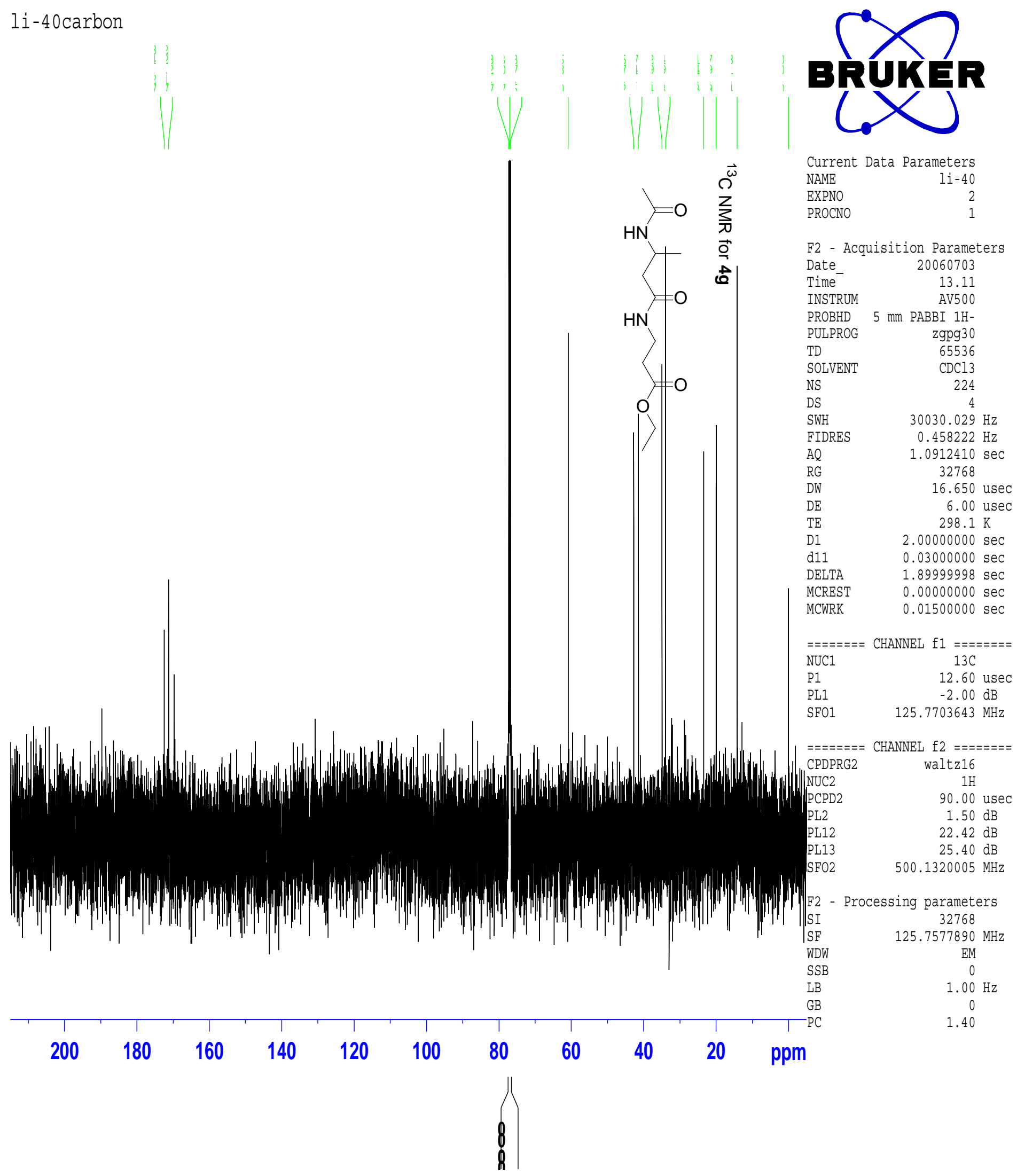